
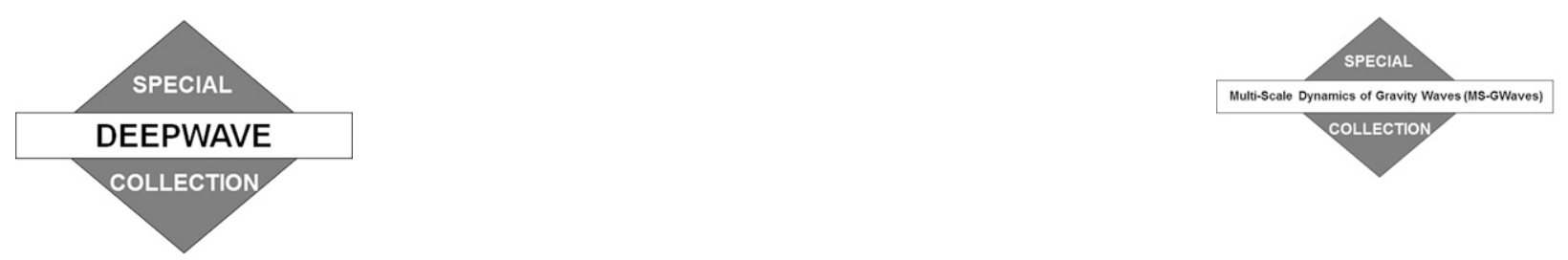

\title{
¿Stratospheric Gravity Waves Excited by a Propagating Rossby Wave TrainA DEEPWAVE Case Study
}

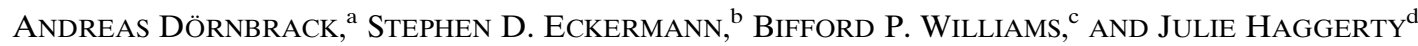 \\ ${ }^{a}$ DLR Oberpfaffenhofen, Institute of Atmospheric Physics, Weßling, Germany \\ ${ }^{\mathrm{b}}$ Space Science Division, U.S. Naval Research Laboratory, Washington, D.C. \\ ${ }^{\mathrm{c}}$ Boulder GATS, Inc., Boulder, Colorado \\ ${ }^{\mathrm{d}}$ National Center for Atmospheric Research, Boulder, Colorado
}

(Manuscript received 3 March 2021, in final form 21 November 2021)

\begin{abstract}
Stratospheric gravity waves observed during the DEEPWAVE research flight RF25 over the Southern Ocean are analyzed and compared with numerical weather prediction (NWP) model results. The quantitative agreement of the NWP model output and the tropospheric and lower-stratospheric observations is remarkable. The high-resolution NWP models are even able to reproduce qualitatively the observed upper-stratospheric gravity waves detected by an airborne Rayleigh lidar. The usage of high-resolution ERA5 data-partially capturing the long internal gravity wavesenabled a thorough interpretation of the particular event. Here, the observed and modeled gravity waves are excited by the stratospheric flow past a deep tropopause depression belonging to an eastward-propagating Rossby wave train. In the reference frame of the propagating Rossby wave, vertically propagating hydrostatic gravity waves appear stationary; in reality, of course, they are transient and propagate horizontally at the phase speed of the Rossby wave. The subsequent refraction of these transient gravity waves into the polar night jet explains their observed and modeled patchy stratospheric occurrence near $60^{\circ} \mathrm{S}$. The combination of both unique airborne observations and high-resolution NWP output provides evidence for the one case investigated in this paper. As the excitation of such gravity waves persists during the quasi-linear propagation phase of the Rossby wave's life cycle, a hypothesis is formulated that parts of the stratospheric gravity wave belt over the Southern Ocean might be generated by such Rossby wave trains propagating along the midlatitude waveguide.
\end{abstract}

KEYWORDS: Gravity waves; Instability; Rossby waves; Stratospheric circulation; Aircraft observations; Numerical analysis/modeling; Reanalysis data

\section{Introduction}

There are only a few airborne observations of middleatmospheric gravity waves in the Southern Hemisphere (Fritts et al. 2016; Rapp et al. 2021). Research flights to latitudes south of $60^{\circ} \mathrm{S}$ are especially rare (e.g., Tuck et al. 1989; Parish and Bromwich 1989; Carli et al. 2000). These early airborne campaigns were mainly motivated by ozone research (Tuck et al. 1997; Tuck 2021) but also documented mesoscale temperature fluctuations (Gary 2008), gravity waves (Bacmeister et al. 1990), and turbulence (Tuck 2008). One of the more recent research flights was conducted during the Deep Propagating Gravity Wave Experiment (DEEPWAVE) experiment in July 2014 using the instrumented NSF/NCAR Gulfstream $\mathrm{V}$ research aircraft from an operating base in Christchurch,

¿Denotes content that is immediately available upon publication as open access.

Corresponding author: Andreas Dörnbrack, andreas.doernbrack@ dlr.de
New Zealand (Fritts et al. 2016). The DEEPWAVE research flight 25 (RF25) went straight southwest from New Zealand to about $63^{\circ} \mathrm{S}$ and returned along the same path back, see Fig. 1 . The scientific objectives of this survey flight into the atmospheric flow above the Southern Ocean were based on operational forecasts by a suite of high resolution numerical weather prediction (NWP) models (Fritts et al. 2016, their Table 3) and were formulated as follows prior to the actual flight:

- to sample a region near Macquarie Island where forecasts suggested strong gravity wave generation and deep propagation from an exit and splitting region of the tropospheric midlatitude jet, here, called the polar front jet,

- to investigate evidence in some global model forecasts that an anomalously strong $\left(>140 \mathrm{~m} \mathrm{~s}^{-1}\right)$ stratopause jet, pushed poleward of its usual climatological location over Christchurch, decelerated rapidly south of New Zealand and spontaneously generated long-wavelength gravity waves with approximately zonally aligned phase fronts in the upper stratosphere, and

- to search for possible orographic gravity wave signatures associated with southeasterly flow over Auckland Island. 

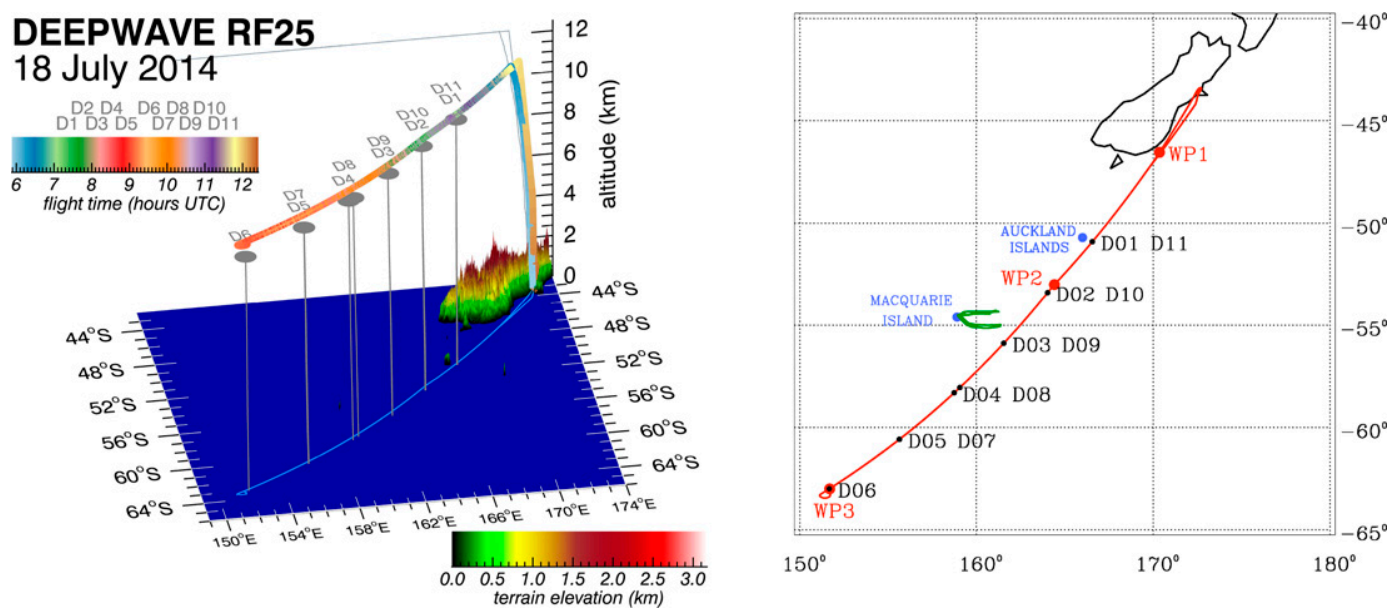

FIG. 1. (left) 3D and (right) top view of the trajectory of the GV research flight RF25 on 18 Jul 2014, showing the launch positions of the 11 dropsondes (marked by D1-D11) and the waypoints WP1, WP2, and WP3. Flight time and terrain elevation of the remote South Island of New Zealand are indicated in the left panel. The total flight distance is about 3100 nautical miles. The green lines starting at Macquarie Island are the trajectories of the seven radiosondes launched from 0000 UTC 17 Jul to 0000 UTC 19 Jul 2014.

At about $60^{\circ} \mathrm{S}$, the airborne Rayleigh lidar observed periodic along-track stratospheric temperature perturbations due to gravity waves inside and above the polar night jet (PNJ). Stratospheric temperature perturbations derived subsequently from the European Centre for Medium-Range Weather Forecasts (ECMWF) reanalysis version 5 (ERA5; Hersbach et al. 2020) revealed extensive regions of gravity wave activity in the reanalysis in and around the southernmost part of the flight track poleward of $55^{\circ} \mathrm{S}$. The existence of these stratospheric gravity waves was validated by observations of wavelike perturbations in thermal infrared nadir satellite radiances over the Southern Ocean that were intercepted by the RF25 flight (Eckermann et al. 2019, their Fig. 18f).

Middle-atmospheric gravity waves at these latitudes may have particularly profound large-scale and climate impacts during austral winter and spring. Localized pockets of gravity wave activity at the vortex edge can form stratospheric ice particles homogeneously that in turn provide nucleation sites for downstream growth of nitric acid trihydrate (NAT) particles. This process can rapidly fill out the vortex edge with growing NAT particles, which will eventually grow large enough to sediment out to denitrify the stratosphere, preconditioning the vortex for rapid ozone loss when sunlight returns in spring (Höpfner et al. 2006). The springtime breakup of the Antarctic vortex typically occurs too late in climate-chemistry models due to an excessively strong vortex, and it has been hypothesized that "missing" gravity wave drag at about $60^{\circ} \mathrm{S}$ in these models may be the source of these problems (e.g., McLandress et al. 2012; Garcia et al. 2017; Garfinkel and Oman 2018).

There are a number of different sources proposed for the non-orographic gravity wave activity that occurs within vortex-edge winds over regions of the Southern Ocean far from orography (e.g., Sato et al. 2012; Alexander and Grimsdell
2013; Hendricks et al. 2014; Hindley et al. 2015; Alexander et al. 2016). As the DEEPWAVE RF25 was far away from dominant orographic sources (see Fig. 1), this paper deals with potential mechanisms of non-orographic gravity wave excitation relevant to stratospheric gravity wave dynamics in this interesting $60^{\circ} \mathrm{S}$ latitude belt.

As a result of a detailed analysis of the airborne observations and the ERA5 meteorological analysis fields, we propose a new mechanism-not previously discussed or consideredthat could contribute to the widespread occurrence of stratospheric gravity waves over the Southern Ocean. In this way, the results of this study might provide connections to the earlier explanations related to non-orographic gravity wave sources in the troposphere by Hendricks et al. (2014); Alexander et al. (2016); Plougonven et al. (2015, 2017).

Traditionally, non-orographic sources are classified as exclusively convective or jet related. Convective generation typically focuses on depths of deep diabatic heating layers that efficiently radiate gravity waves. Jet generation typically focuses on higher-order imbalance within rapidly evolving baroclinic jet exit regions that is restored by spontaneous gravity wave emission. In practice, the two processes can be intertwined: for example, intense baroclinic storms in the Southern Ocean are characterized by both deformational imbalances and moist stratiform convection, both of which can generate gravity waves. Indeed, modern studies of jetrelated gravity waves from baroclinic storms include diabatic moist processes in the modeling (e.g., Wei and Zhang 2014; Plougonven et al. 2015).

Here we focus on a generation mechanism for stratospheric gravity waves that shares similarities with orographic generation: the so-called moving mountain mechanism as proposed by Pfister et al. (1993) treated convective anvils as presenting a time-varying obstacle-like surface to the overlying 
stratospheric flow, which excites gravity waves much like flow over stationary surface terrain. In this work, we propose that the deformed extratropical tropopause associated with baroclinic processes provides a similar obstacle-type forcing to the overlying flow. In a climatological sense, the extratropical tropopause is characterized by a pronounced inversion layer, see Birner et al. (2002), that may act as an interface for the overlaying stratospheric flow. Already Clark et al. (1986) proposed a similar concept for generating gravity waves by the tropospheric flow over a capping inversion of the atmospheric boundary layer.

Considering the regular appearance of propagating Rossby waves at middle latitudes in the Southern Hemisphere, the meandering tropopause jet results in tropopause undulations that travel east with the phase speed of the Rossby waves. Stationary patterns like blocks are rare in the Southern Hemisphere. Due to the relative motion of the eastward-propagating tropopause undulations and the increasing strong westerly stratospheric flow above, internal gravity waves are excited and can easily penetrate into the middle atmosphere during austral winter. Moreover, as these "tropopause waves" are excited at middle latitudes $\left(50^{\circ}-55^{\circ} \mathrm{S}\right)$ and the orientation of the tropopause depressions is rarely exactly north-south, the wave vector is inclined with respect to the predominantly westerly flow above. Therefore, these waves are refracted meridionally into the core of the PNJ (Dunkerton 1984) that is commonly located south at around $60^{\circ} \mathrm{S}$. This refraction of internal gravity waves into the Southern Hemispheric PNJ has been described by a number of publications for stationary mountain waves (e.g., Preusse et al. 2002; Sato et al. 2012; Ehard et al. 2017; Jiang et al. 2019; Hindley et al. 2020). The combination of both processes - the excitation of transient gravity waves and their meridional and vertical propagation - can lead to zonally elongated, widespread stratospheric gravity wave activity all the way around the Southern Ocean, since the baroclinic systems that produce these dynamics regularly traverse the Southern Ocean.

The objectives of this work are the following. First, the unique airborne observations covering the atmosphere from the surface to about $60-\mathrm{km}$ altitude are analyzed. Comparison with data from NWP models leads to a characterization of atmospheric flow in the troposphere and stratosphere. In particular, airborne lidar observations will be used to detect and analyze stratospheric gravity waves over the Southern Ocean. The second objective is to use the latest ECMWF analyses and reanalyses to elucidate the sources of the stratospheric gravity waves. To this end, vertical wave energy fluxes will be correlated with various parameters that define the tropospheric and upper-level flow. This analysis leads to the hypothesis that the most likely source of the observed and modeled stratospheric gravity waves is related to tropopause depressions associated with eastward-propagating Rossby waves.

In this way, the paper proceeds in the following steps. Section 2 introduces the DEEPWAVE RF25 as well as the observational and model data used for this study. Section 3 overviews the atmospheric flow conditions in the troposphere and stratosphere during an extended period of time when
TABLE 1. Partition of the GV flight legs as depicted in Fig. 1.

\begin{tabular}{llccc}
\hline \hline & Way points & Time (s) & UTC & $\begin{array}{c}\text { Length } \\
(\mathrm{km})\end{array}$ \\
\hline Outbound leg & $\mathrm{WP1:} 46.5^{\circ} \mathrm{S}, 170.4^{\circ} \mathrm{E}$ & 23000 & 0623 & - \\
& $\mathrm{WP2}: 53.0^{\circ} \mathrm{S}, 164.4^{\circ} \mathrm{E}$, & 26566 & 0723 & 842 \\
& $\mathrm{WP} 3: 63.0^{\circ} \mathrm{S}, 151.7^{\circ} \mathrm{E}$ & 32593 & 0903 & 1336 \\
& & & & \\
Inbound leg & $\mathrm{WP3}: 63.0^{\circ} \mathrm{S}, 151.7^{\circ} \mathrm{E}$ & 33145 & 0912 & - \\
& $\mathrm{WP2:} 53.0^{\circ} \mathrm{S}, 164.4^{\circ} \mathrm{E}$ & 38667 & 1045 & 1336 \\
& $\mathrm{WP1:} 46.2^{\circ} \mathrm{S}, 170.7^{\circ} \mathrm{E}$ & 42480 & 1148 & 842 \\
\hline
\end{tabular}

RF25 took place as well. Section 4 presents the stratospheric gravity waves based on the airborne Rayleigh lidar observations and the ERA5 data. Section 5 evaluates different hypotheses of the origins of the observed waves and substantiates our proposed mechanism. Finally, section 6 concludes this paper. Additionally, the appendix contains comparisons of the ECMWF data with airborne and radiosonde observations providing confidence in the realism of the simulated model fields.

\section{Data}

The National Science Foundation (NSF)-National Center for Atmospheric Research (NCAR) Gulfstream V (GV) research flight RF25 during DEEPWAVE was conducted on 18 July 2014 from takeoff at 0552 UTC (1752 New Zealand standard time, NZST) until landing at 1225 UTC (0025 NZST 19 July 2014). The flight had a total duration of about $6.5 \mathrm{~h}$. Since the flight period was centered approximately at 0900 UTC, this time will be used for further presentation of the meteorology. The GV-flight track is plotted in Fig. 1. The flight operated at FL400, i.e., at $40 \mathrm{kft}$, so that the cruise altitude was about $12 \mathrm{~km}$ throughout. The outbound and inbound legs of the flight track passed close to the southeast of Auckland Island and downstream of Macquarie Island. The flight then extended to the south and west to intercept the predicted tropospheric jet exit region. For the following analysis, the flight track is divided into an outbound and an inbound leg between waypoints WP1 and WP3 each separated into north and south segments by intermediate waypoint WP2, see Table 1.

All onboard instruments operated without any reported failure. Here, four measurement systems are included into the analysis of this case. The flight-level in situ data (UCAR/ NCAR-Earth Observing Laboratory 2015a) are used to estimate the wave energy and momentum fluxes along the flight path, see Smith et al. (2016) and Cooper et al. (2016) for the specifications of the accuracy of the measured quantities UIC, VIC, WIC, WSC, WDC, ATX, and THETA, representing the velocity components $u, v$, and $w$, the horizontal wind speed $V_{H}$ and wind direction $\alpha_{H}$, and the potential temperature $\Theta$, respectively.

As marked by the dots in Fig. 1, 11 dropsondes (UCAR/ NCAR-Earth Observing Laboratory 2016) were released at equally spaced great circle distances between Auckland Island 
and the southernmost way point WP3 of the mission at about $63^{\circ} \mathrm{S}$. The northernmost D01 and D11 soundings were thought to define the flow forcing environment for possible orographic gravity waves observed from the flyby of Auckland Island. It should be noted that these dropsonde data were ingested as initial conditions into both the Navy and ECWMF operational global model forecasts and their subsequent reanalysis products on this day (Eckermann et al. 2018, their Fig. 8).

The Rayleigh lidar was a new facility instrument built at Boulder GATS, Inc., for the NSF/NCAR Gulfstream V (Williams 2015). The laser is a diode-pumped Nd:YLF Photonics DS20-351 generating $5 \mathrm{~W}$ at a $351-\mathrm{nm}$ wavelength and a $1-\mathrm{kHz}$ pulse repetition rate. The telescope is a 305 -mm-diameter f/4 Newtonian design supported under the aircraft vertical viewport by a plate between the two lidar racks. The fibercoupled detector is a $50 \%$ quantum efficiency, low noise photo multiplier tube with a $0.5-\mathrm{nm}$ FWHM interference filter. The returned signal profiles are recorded at 1-s-temporal and 37.5-m-altitude resolutions. Here, 180-s mean values interpolated on a regular 1-km grid in the vertical are used.

The atmospheric density profile was calculated from the lidar signals, taking into account the geometric factors, and then integrating the density down to obtain a temperature profile (Hauchecorne and Chanin 1980). For the start temperature, the ECMWF model temperature at $71 \mathrm{~km}$ was interpolated to the aircraft time and position. The temperature profile becomes independent of the assumed starting temperature at 1-2 scale heights below $71 \mathrm{~km}$. This calculation assumes that there is no aerosol backscatter, so results starting from 20 to $25 \mathrm{~km}$ and up to about $60-\mathrm{km}$ altitude are investigated. At $40-\mathrm{km}$ altitude the temperature error is about $1 \mathrm{~K}$, at $60-\mathrm{km}$ altitude the error increases to about $5 \mathrm{~K}$.

The Microwave Temperature Profiler (MTP) (UCAR/ NCAR-Earth Observing Laboratory 2015b) provides reliable absolute and potential temperatures with a layer $\pm 3-\mathrm{km}$ thick centered around the flight level (Gary 1989; Denning et al. 1989; Mahoney and Denning 2009). As indicated on the EOL web page, ${ }^{1}$ the retrieval quality is reasonable for RF25. Some of the MTP-dropsonde comparisons show significant bias at lower altitudes. We discuss this issue in greater depth in the appendix.

In addition to the airborne measurements, seven radiosonde ascents (routine soundings at 0000 and 1200 UTC from 17 to 19 July, and extra soundings at 0600 and 0900 UTC 18 July 2014) from Macquarie Island (Joyce 2014) are used to compare the meteorological analyses with the vertical profiles which went up to about $30-\mathrm{km}$ altitude. These observations were also assimilated by the NWP centers.

Operational analyses of the integrated forecast system (IFS) of the ECMWF are used to provide meteorological data to characterize the atmospheric environment; these data will be denoted by HRES IFS. ${ }^{2}$ The model fields of the IFS cycle 40r1 operating at this time have a horizontal resolution of

\footnotetext{
${ }^{1} \mathrm{https} / /$ www.eol.ucar.edu/content/summary-mtp-results-deepwave.

${ }^{2}$ The atmospheric model high-resolution deterministic 10 days forecasts, https://www.ecmwf.int/en/forecasts/datasets/set-i.
}

about $16 \mathrm{~km}\left(\mathrm{~T}_{\mathrm{L}} 1279\right.$ where the subscript $\mathrm{L}$ stand for the linear Gaussian grid) and 137 vertical model levels. The model top of the IFS was located at $0.01 \mathrm{hPa}$. Additionally, most recent reanalysis data, ERA5 (Hersbach et al. 2020), are used for the selected time period. ERA5 is based on the IFS cycle 41r2 and provides hourly estimates of atmospheric variables as analysis fields. The data cover Earth on a $30-\mathrm{km}$ grid $\left[\mathrm{T}_{\mathrm{C}} 639\right.$, where the subscript $\mathrm{C}$ denotes a triangular spectral truncation with 639 wavenumbers associated with a cubic reduced Gaussian spectral transform grid, see Wedi (2014); Malardel and Wedi (2016)].

ERA5 temperature perturbations $T_{\text {ERA5 }}^{\prime}$ were calculated as differences between the temperatures from fields retrieved at full spectral resolution and spectrally truncated fields containing contributions from all horizontal wavenumbers up to 106 interpolated on the same $0.28125^{\circ} \times 0.28125^{\circ}$ regular latitude-longitude grid. The total horizontal wavenumber 106 is selected as it can be used to differentiate between synopticscale and mesoscale modes as due to inertia-gravity waves (Žagar et al. 2017; Schumann 2019). Therefore, positive and negative $T_{\mathrm{ERA} 5}^{\prime}$ values can be associated with troughs and crests, i.e., with the phases of the stratospheric gravity waves. As the effective useful horizontal resolution of these reanalyses is about 90-150 km [about 3-5 times the grid spacing, see discussions in Abdalla et al. (2013); Malardel et al. (2016)], only gravity waves with wavelengths larger than about $150 \mathrm{~km}$ can be resolved.

In addition to the IFS data, recently published research Navy Global Environmental Model [NAVGEM; Hogan et al. (2014)] reanalysis runs for the 2014 austral winter are used (Eckermann et al. 2018). Two hybrid-4DVar (HYBRID) runs were conducted differing in the horizontal resolutions. The run HYBRID119 yields a global resolution of $1^{\circ} \times 1^{\circ}$ and the run HYBRID425 yields grid cells at the latitude of Christchurch $\left(\approx 45^{\circ} \mathrm{S}\right)$ of $\approx 0.38^{\circ}$ in longitude and $\approx 0.28^{\circ}$ in latitude. Both runs were designed to provide atmospheric data from the surface to $100-\mathrm{km}$ altitude capturing the large-scale structure affecting gravity wave generation, propagation and breakdown processes (HYBRID119) and partially resolving gravity wave dynamics (HYBRID425).

\section{Atmospheric flow conditions}

\section{a. Tropospheric flow}

The surface and frontal analysis of the ERA5 data as presented for the mean sea level pressure (MSLP) and the equivalent potential temperature $\Theta_{e}$ at $850 \mathrm{hPa}$ and valid at 0900 UTC 18 July 2014 in Fig. 2 reveals two deep surface lows (core pressure less than $980 \mathrm{hPa}$ ) around and south of $60^{\circ} \mathrm{S}$ (Fig. 2a). The elongated cold front northeast of Macquarie Island (Fig. 2b) was associated with the eastern low located at around $160^{\circ} \mathrm{E}$ (Fig. 2a). The GV flight track crossed this northeastward-propagating frontal zone both on the outbound and inbound legs. The tropospheric flow in their southern segments was dominated by polar air masses whereas the northern segments belonged to subtropical air. The meridional surface pressure difference from Macquarie Island to the lows in the storm track north of the coast of Antarctica 

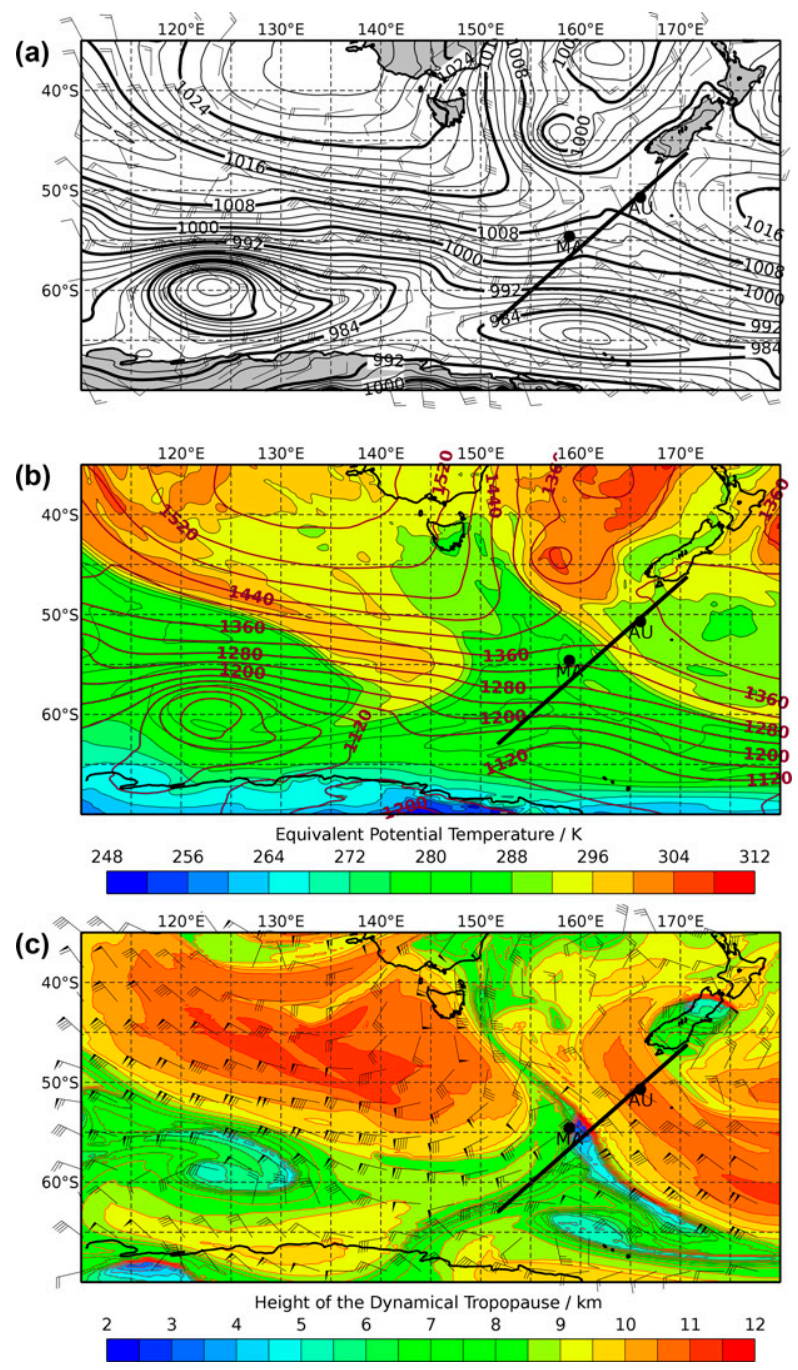

FIG. 2. (a) Mean sea level pressure (hPa, solid lines) and horizontal wind ( $\mathrm{m} \mathrm{s}^{-1}$, barbs) at 10-m altitude. (b) Equivalent potential temperature (K, color shaded) at the $850-\mathrm{hPa}$ pressure level. (c) Altitude ( $\mathrm{km}$, color shaded) of and horizontal wind $\left(\mathrm{m} \mathrm{s}^{-1}\right.$, barbs) at the dynamical tropopause defined as the 2-PVU level. Valid time: 0900 UTC 18 Jul 2014. Data: ERA5 on a $0.28125^{\circ}$ regular latitude-longitude grid. The letters AU and MA mark the locations of Auckland Islands and Macquarie Island. The black solid line displays the flight track of the GV aircraft.

reached about $30 \mathrm{hPa}$ and led to strong zonal near-surface winds over large portions of the Southern Ocean (Fig. 2a). As the frontal systems propagated about $600 \mathrm{~km}$ eastward in the course of RF25 the tropospheric flow varied both in strength and direction during the research flight.

The Southern Hemispheric storm track is characterized by synoptic-scale transient eddies that manifest themselves as a regular sequence of eastward-propagating high and low pressure systems (e.g., Trenberth 1991; Hoskins and Hodges 2005; Schultz et al. 2019). In the upper troposphere these transients appear in form of Rossby wave trains, i.e., Rossby waves for which the amplitude envelope is modulated with longitude (e.g., Madden 2007; Glatt et al. 2011). The Rossby waves owe their existence to the zonal waveguide that appears as a sharp meridional potential vorticity gradient (e.g., Martius et al. 2010; Wirth et al. 2018). Rossby waves can be visualized by tropopause maps depicting potential temperature $\Theta_{\text {tropo }}$, pressure $p_{\text {tropo }}$, or geopotential height $z_{\text {tropo }}$ on surfaces with a constant potential vorticity of 2 PVU ( $\left.1 \mathrm{PVU}=10^{-6} \mathrm{~K} \mathrm{~kg}^{-1} \mathrm{~m}^{2} \mathrm{~s}^{-1}\right)$ (Morgan and Nielsen-Gammon 1998). Figure 2c shows a deep tropopause depression associated with the upper-level front that propagated northeastward during RF25. Along the flight track, the analyzed tropopause height varied from about $12 \mathrm{~km}$ southwest of New Zealand down to about $5 \mathrm{~km}$ inside the depression. Figure 3 a illustrates the eastward propagation of the tropospheric weather systems by means of Hovmöller diagrams for the meridional wind component $v_{\text {tropo }}$. Unlike in the classical paper by Hovmöller (1949), here, the $v$ field is visualized on the 2-PVU surface and the depth of the tropopause for values $z_{\text {tropo }} \leq 8 \mathrm{~km}$ is included in all panels of Fig. 3. Both $v_{\text {tropo }}$ and $z_{\text {tropo }}$ propagate quasi-linearly eastward with a phase speed of about $17 \mathrm{~m} \mathrm{~s}^{-1}$ as estimated from Fig. 3a. During the life cycle of the Rossby wave the amplitude of both quantities is modulated and a more extended plot would show the whole Rossby wave train.

Naturally for upper-level fronts, thermal gradients appear along the tropopause depression (Reed and Danielsen 1958). In Figs. 3b,c, the magnitude of the gradient of the potential temperature $\left|\nabla \Theta_{\text {tropo }}\right|$ and the thermal front parameter

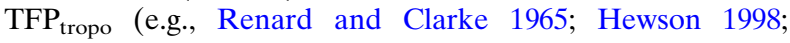
Thomas and Schultz 2019) defined as

$$
\mathrm{TFP}_{\text {tropo }}=-\nabla\left|\nabla \Theta_{\text {tropo }}\right| \cdot \frac{\nabla \Theta_{\text {tropo }}}{\left|\nabla \Theta_{\text {tropo }}\right|},
$$

which are visualized to quantify the strength of the upperlevel front. Here, we largely follow the plotting conventions proposed by Thomas and Schultz (2019) and the negative sign in Eq. (1) ensures that the warm side of the frontal zone has $\mathrm{TFP}_{\text {tropo }}>0$ (Hewson 1998). The magnitude of $\left|\nabla \Theta_{\text {tropo }}\right|$ follows the contour lines of $z_{\text {tropo }}$ and achieves values of $6 \mathrm{~K}$ $(100 \mathrm{~km})^{-1}$. For surface fronts, values larger $4 \mathrm{~K}(110 \mathrm{~km})^{-1}$ are considered as moderate baroclinic zones (Thomas and Schultz 2019). Thus, during the life cycle of the Rossby wave, the strength of the thermal front is also modulated. This is also visible in $\mathrm{TFP}_{\text {tropo }}$ : the warm front is located east to the tropopause depression and values of $\mathrm{TFP}_{\text {tropo }}>3 \mathrm{~K}(100 \mathrm{~km})^{-2}$ indicate strong frontal gradients. More importantly, both quantities $\left|\nabla \Theta_{\text {tropo }}\right|$ and $\mathrm{TFP}_{\text {tropo }}$ follow the phase propagation of the progressing Rossby wave and, therefore, can be used to quantify its evolution in the same way as the depth of the tropopause $z_{\text {tropo. }}$

Figure 4 shows the analyzed horizontal wind $V_{H}$ and geopotential height $Z$ at $1,10,100$, and $300 \mathrm{hPa}$, respectively, valid at 0900 UTC 18 July 2014. The upper-tropospheric wind at $300 \mathrm{hPa}$ is characterized by the slightly poleward tilted polar front jet at about $50^{\circ} \mathrm{S}$ (Fig. 4d). Near Macquarie Island, the polar front jet slows sharply and is deflected to the north and Tasmania. East of Macquarie Island, the entrance region of another branch of the polar front jet moves farther east and 

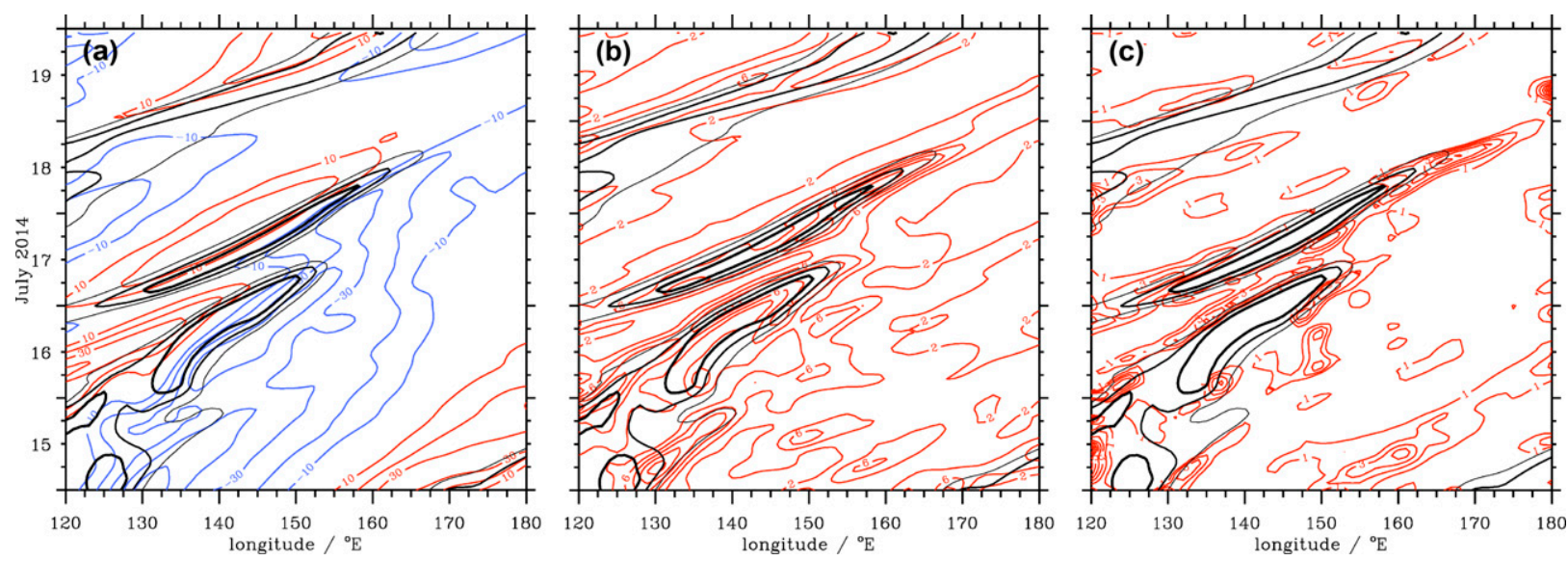

FIG. 3. Hovmöller plots of selected quantities taken at the dynamical tropopause defined as the 2-PVU surface and along $55^{\circ} \mathrm{S}$. (a) Meridional wind component $v_{\text {tropo }}\left(\mathrm{m} \mathrm{s}^{-1}\right.$, red and blue contour lines with an increment of $\left.10 \mathrm{~m} \mathrm{~s}^{-1}\right)$, (b) the magnitude of the gradient of the potential temperature $\left|\nabla \Theta_{\text {tropo }}\right|\left[\mathrm{K}(100 \mathrm{~km})^{-1}\right.$, red contour lines with an increment of $\left.2 \mathrm{~K}(100 \mathrm{~km})^{-1}\right]$, and (c) the thermal front parameter $\mathrm{TFP}_{\text {tropo }}\left[\mathrm{K}(100 \mathrm{~km})^{-2}\right.$, red and blue contour lines with an increment of $\left.1 \mathrm{~K}(100 \mathrm{~km})^{-2}\right]$ as defined by Eq. (1). The three black contour lines represent the height of the dynamical tropopause $z_{\text {tropo }}$ of 6,7 , and $8 \mathrm{~km}$ (thick to thin lines) in each panel. Data: 1-hourly ERA5 data averaged over a latitudinal band from $52.5^{\circ}$ to $57.5^{\circ} \mathrm{S}$ and interpolated on a $1^{\circ}$ regular longitude grid. 17 to 19 July 2014.

\section{b. Stratospheric flow}

poleward, where the flow strengthens again with $V_{H}$ exceeding $80 \mathrm{~m} \mathrm{~s}^{-1}$. Animations of the $300 \mathrm{hPa}$ winds (not shown) document the intensification of the eastern jet (whose entrance region just touches the RF25 track in Fig. 4d) and the eastward propagation of the overall flow pattern from

At $100 \mathrm{hPa}$, the polar night jet is located farther to the south compared to the tropospheric jet, cf. Fig. 4c. A similar
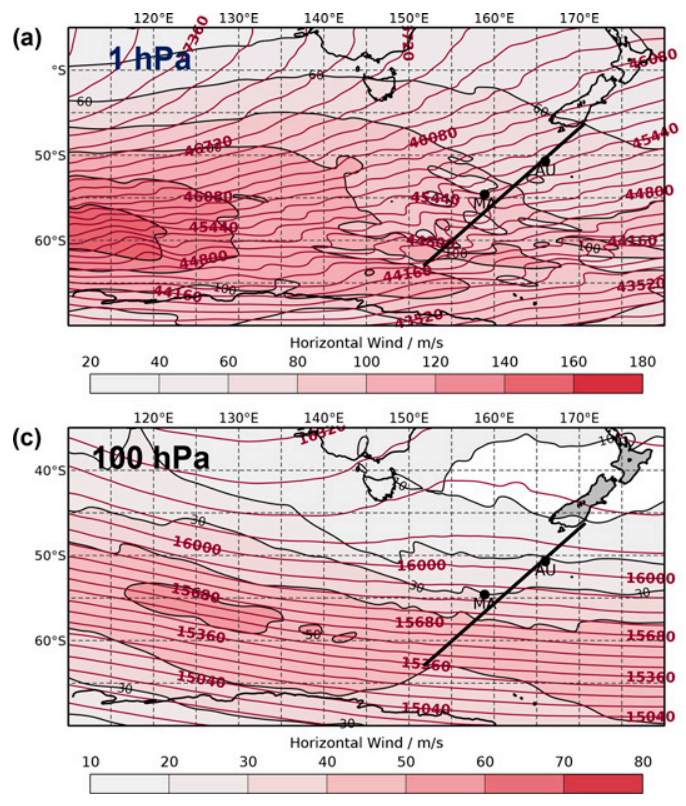

zonal pattern of stratospheric flow dominates also at $10 \mathrm{hPa}$ where $V_{H}$ more than doubled (Fig. 4b) compared to $100 \mathrm{hPa}$. In the climatological mean for July, the core of PNJ at $10 \mathrm{hPa}$ is located north of $60^{\circ} \mathrm{S}$. Thus, the poleward displacement of the maximum horizontal winds indicates the tilt of the Antarctic stratospheric polar vortex due to planetary wave activity during this time, see Gisinger et al. (2017); Eckermann et al. (2018). The poleward concentration of $Z$ contour lines marks the strong winds of the PNJ at the edge of the polar vortex. In addition, the large stratospheric anticyclone at
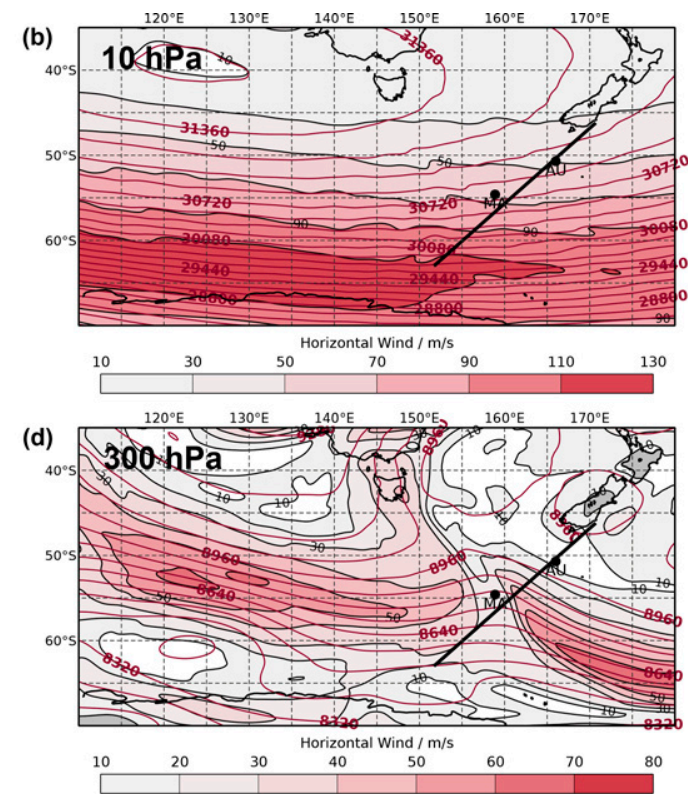

FIG. 4. Horizontal wind ( $\mathrm{m} \mathrm{s}^{-1}$, color shaded) and geopotential height ( $\mathrm{m}$, burgundy solid lines) at (a) 1, (b) 10, (c) 100, and (d) $300 \mathrm{hPa}$ at 0900 UTC $18 \mathrm{Jul} 2014$. Data: ERA5 on a $0.28125^{\circ}$ regular latitude-longitude grid. Please note the different color bars for the individual panels. 

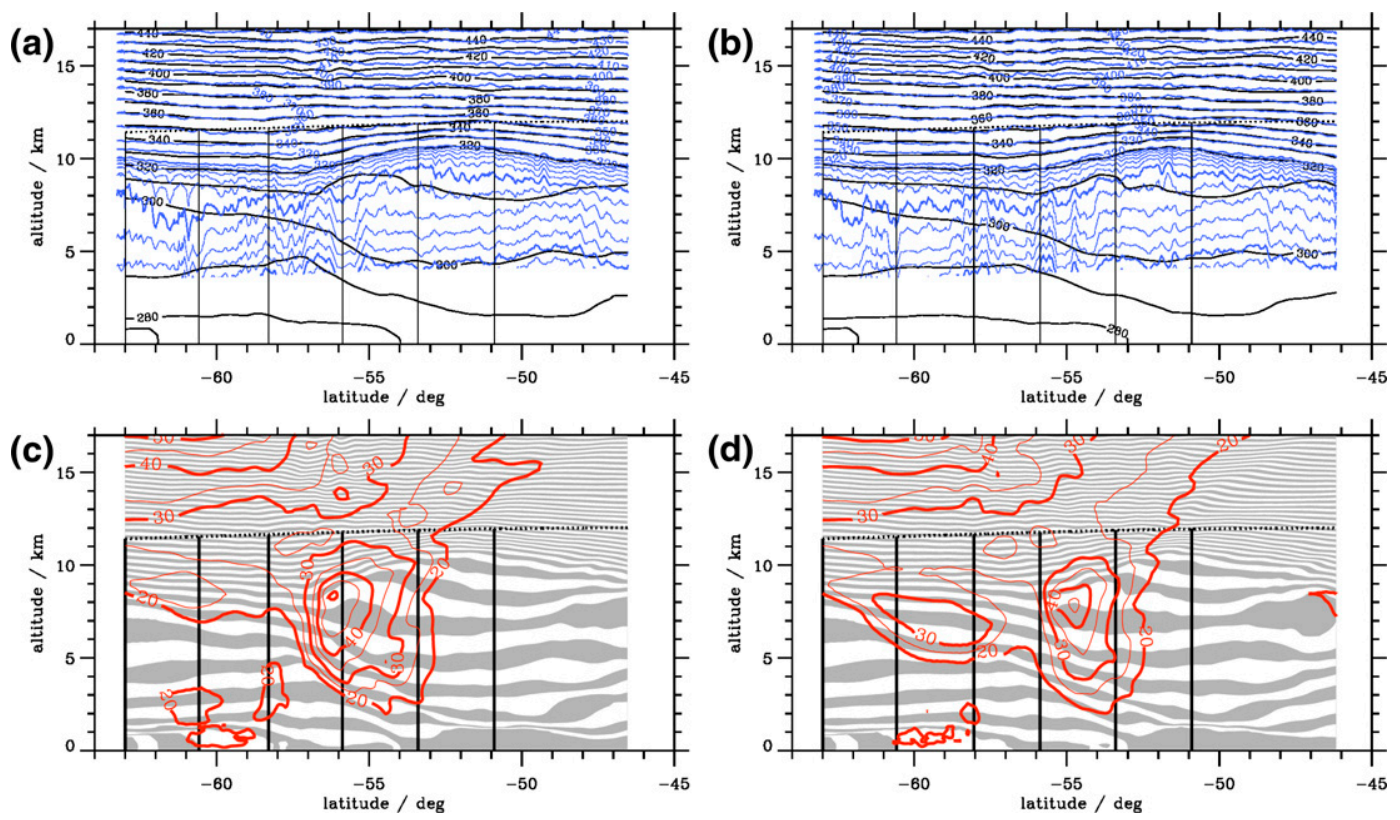

FIG. 5. Potential temperature iso-contours from the GV MTP retrieval (thick blue lines: 10-K increments; thin blue lines: 2-K increments) and from the IFS interpolated analysis and forecast data (black lines) for (a) the outbound leg and (b) the inbound leg of RF25IFS potential temperature in zebra-like contouring (each $5 \mathrm{~K}$ wide) and horizontal wind $\left(\mathrm{m} \mathrm{s}^{-1}\right.$, red contours every $\left.5 \mathrm{~m} \mathrm{~s}^{-1}\right)$ for (c) the outbound leg and (d) the inbound leg. The GV flight altitude and the vertical profiles of the dropsondes along the respective legs are marked in the individual panels.

$10 \mathrm{hPa}$ centered at around $40^{\circ} \mathrm{S}, 120^{\circ} \mathrm{E}$ leads to anomalously large meridional gradients of the zonal wind spanning the whole GV flight track from north to south; cf. Fig. 8 in Gisinger et al. (2017). At this pressure level—and much more dominant at $1 \mathrm{hPa}$ (Fig. 4a)-subsynoptic disturbances are visible in $V_{H}$ as well as in $Z$ that manifest themselves as waves in the respective contour lines. The occurrence of these wavelike undulations goes along with the deceleration of the PNJ at $1 \mathrm{hPa}$ east of about $130^{\circ} \mathrm{E}$.

As a first check of the convincing realism of the numerical IFS simulations, Fig. A1 in the appendix presents a comparison with the seven operational radiosonde profiles launched from Macquarie Island between 16 and 19 July 2014. The agreement between observations and the IFS is excellent; discernible deviations only exist for the 0900 UTC profile on 18 July 2014 for which IFS forecasts with a lead time of $9 \mathrm{~h}$ have been used instead of the operational analyses.

Supporting this, the potential temperature profiles calculated from the retrieved MTP temperature along the out- and inbound legs reveal a fair agreement with the IFS isentropes in immediate vicinity to the flight altitude at $12 \mathrm{~km}$, as shown in Fig. 5. The range of potential temperatures from the MTP as shown in Fig. 5 is limited to values between 295 and $485 \mathrm{~K}$. The vertical distribution of the isentropes indicates a higher tropopause of about $10-11-\mathrm{km}$ altitude in the northern segments of the out- and inbound legs and a lower tropopause of about 8-9-km altitude in the south. In the middle of both legs, a large-scale wavelike deformation of the isentropes dominates north of about $55^{\circ} \mathrm{S}$. Comparing the MTP profiles with the IFS isentropes and winds reveals that this region is associated with the presence of the polar front jet. Consistent with idealized textbook models (Shapiro and Keyser 1990; Shapiro et al. 1999; Schultz et al. 2019), the descent of the tropopause on the poleward side and the analyzed spreading of isentropes to the north (Figs. $5 \mathrm{c}$,d) clearly indicate that the baroclinic zones associated with the upper-level front at about $8-\mathrm{km}$ altitude are slightly shifted to the south compared to the surface front lower down. The location of the exit region of the tropospheric jet at about $60^{\circ} \mathrm{S}$ as well as the weakening winds of the eastern jet due to its eastward propagation are discernible along the inbound leg in Fig. 5d, see also Fig. 4d Visible both in the MTP data as well as in the IFS data are wavelike patterns with small amplitudes extending northward into the stratosphere.

As indicated in the previous section, the MTP retrieval shows deviations to the dropsondes at lower altitudes and, especially, at the southern part of the RF25. The appendix provides a comparison of the retrieved MTP temperature data with selected GV dropsonde profiles. The sufficiently good agreement between MTP temperatures and dropsonde observations degrades drastically for increasing distance from the flight level. Furthermore, the MTP profiles are set off vertically from the observed temperature profiles in the southern portion of the legs. This deviation is most probably due to non-representative a priori temperature profiles far away from reliable radiosondes which are used as initial guesses for the retrieval. Also at larger distances from the aircraft, accuracy of the retrieved temperature profiles decreases since the MTP is most accurate within $\pm 3 \mathrm{~km}$ of flight level (Gary 1989; Denning et al. 1989). The horizontally short fluctuations 

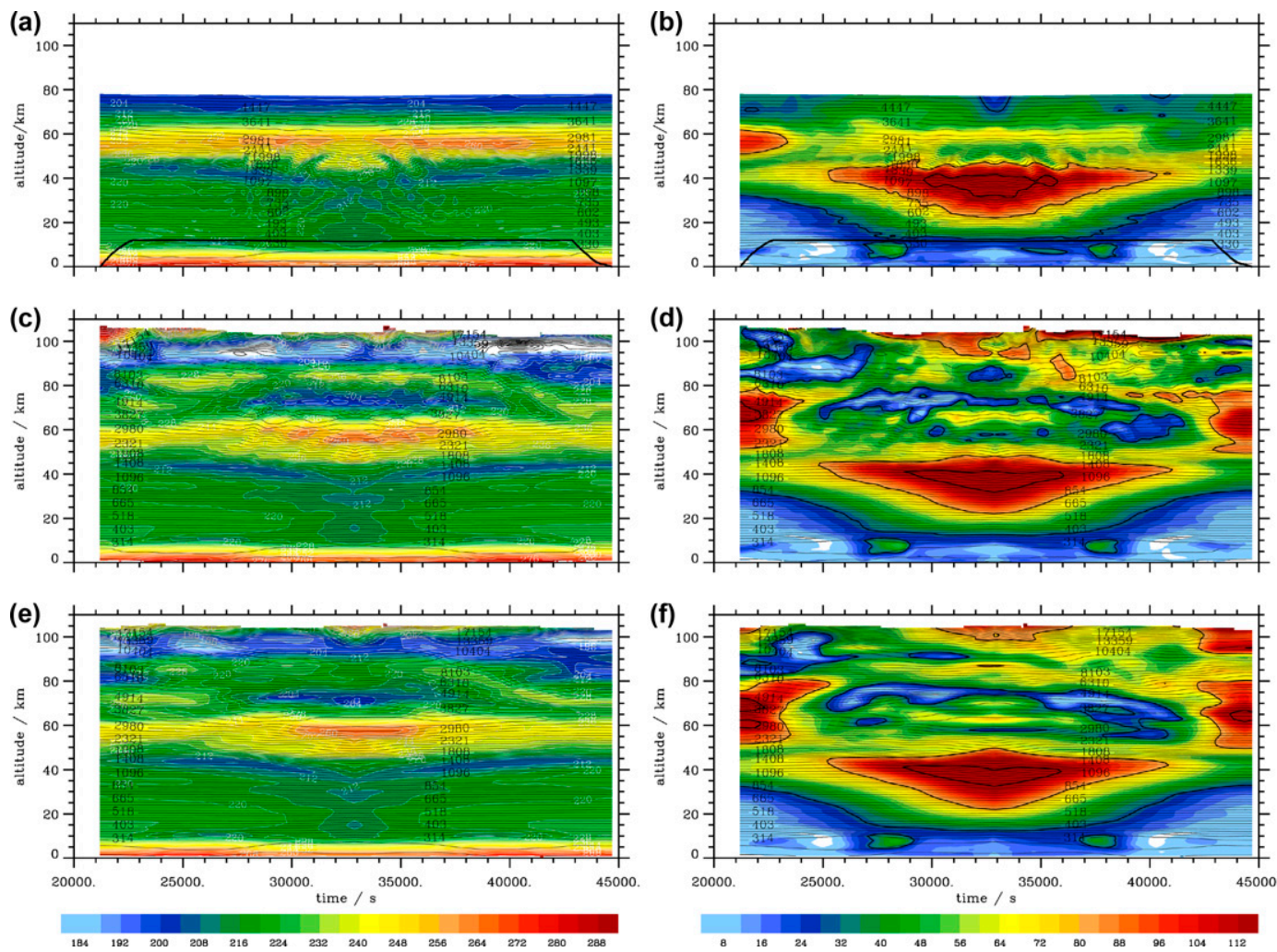

FIG. 6. (a),(c),(e) Absolute temperature (K, color shaded) and (b),(d),(f) horizontal wind ( $\mathrm{m} \mathrm{s}^{-1}$, color shaded, black contour lines at 30, 80, and $120 \mathrm{~m} \mathrm{~s}^{-1}$ ) as function of UTC time since midnight of $18 \mathrm{Jul} 2014$ during RF25. The thin black lines are isentropes in a logarithmic spacing. The thick black lines in (a) and (b) mark the altitude profile of the GV: (a),(b) ECMWF IFS 6-hourly analyses and 1-hourly forecasts and (c),(d) high-resolution and (e),(f) lowresolution versions of the hybrid-4DVAR NAVGEM reanalysis interpolated in space and time on the RF25 flight track of the GV.

in the tropospheric part of the remote sensing MTP observations are not considered here.

Finally, Fig. 6 profiles the absolute temperature and horizontal wind $V_{H}$ along the flight track of RF25 covering the altitude range from the surface to about $100 \mathrm{~km}$ whereby the IFS operational analysis and forecast data top out at $80-\mathrm{km}$ altitude. The 1-hourly IFS data are interpolated in space and time on the 1-s GV positions. The rather symmetric patterns around the southernmost way point WP3 result from the same track coordinates of the out- and inbound legs in a slowly evolving atmospheric flow. The absolute temperature shows the characteristic vertical structure of the winter polar atmosphere: a warm troposphere resides under the cold and stably stratified stratosphere which is topped by the temperature maximum of the stratopause (Figs. 6a,c,e). In the mesosphere, the temperature decreases again. Poleward of New Zealand, $V_{H}$ is dominated by high values greater than $120 \mathrm{~m} \mathrm{~s}^{-1}$ inside the PNJ between 20- and 55-km altitude (Figs. 6b,d,f). Underneath, the out- and inbound legs of the GV crossed the polar front jet $\left(V_{H}>30 \mathrm{~m} \mathrm{~s}^{-1}\right)$ as mentioned before. The mesospheric wind and temperature as well as the small-scale gravity waves at these altitudes are not the subject of this paper, see Pautet et al. (2019).
However, the appearance of the stratospheric split jet near the stratopause (Eckermann et al. 2018) - presenting itself by the $V_{H}$ maxima at around $70-\mathrm{km}$ altitude at the begin and end of RF25-is worth mentioning (Figs. 6d,f). This split-jet structure is the result of the large-amplitude, quasi-stationary wavenumber 1 planetary wave activity in July 2014 displacing the stratospheric PNJ poleward (Gisinger et al. 2017; Eckermann et al. 2018).

The close agreement between the IFS data and the lowand high-resolution hybrid-4DVAR NAVGEM reanalyses documents consistent tropospheric and stratospheric flow features inspiring further confidence in the realism of the NWP models' analysis products. Moreover, both the $V_{H}$ as well as the potential temperature contours by the IFS and the highresolution NAVGEM data show undulations in the upper stratosphere. These wavelike patterns are clearly detectable by inspecting the IFS vertical wind as shown in Fig. 7a and the temperature perturbations $T^{\prime \prime}=T-\langle T\rangle$ (where $\langle T\rangle$ is a 180-s running mean of $T$ ) as shown in Fig. 7b. In the upper stratosphere, poleward curved regular patterns appear in the shape of a lancet arch window due to the symmetry of the flight track. Above the stratopause, the wave amplitude decreases. Below 20-km altitude, Figs. 7a and 7b show no resolved waves 

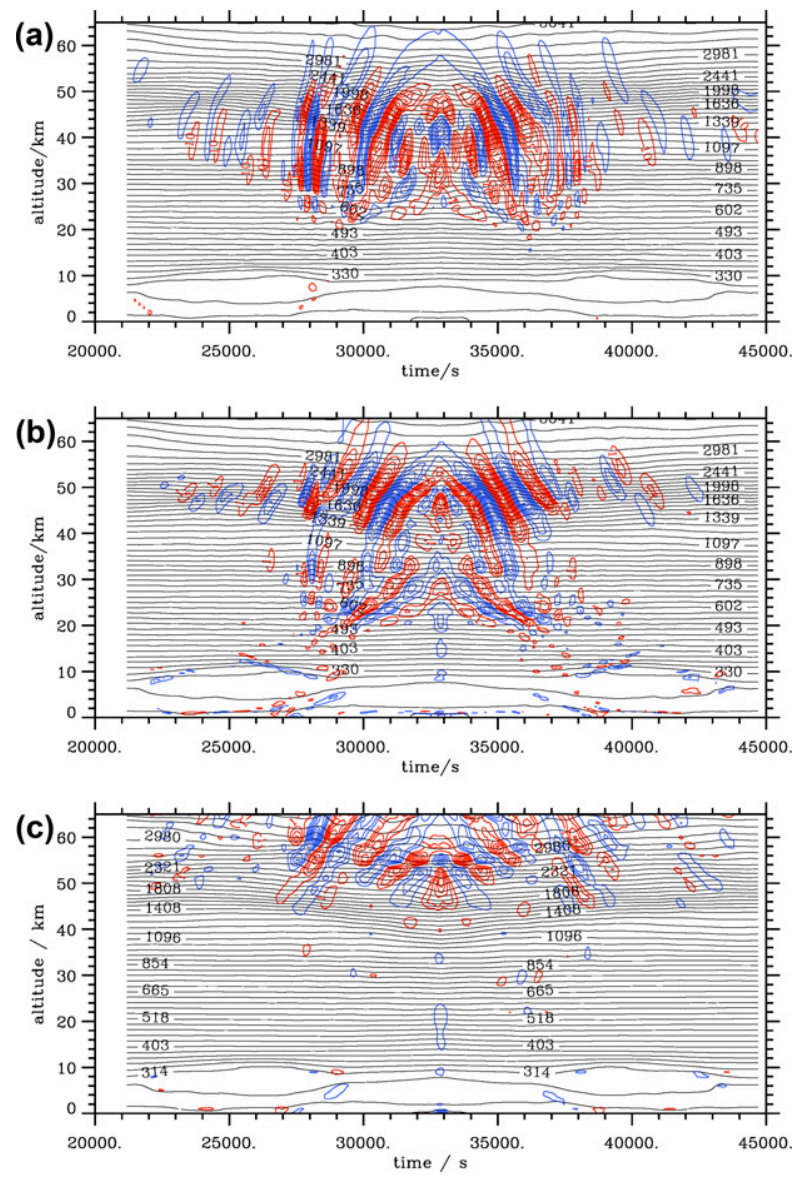

FIG. 7. (a) IFS vertical velocity component $\left(\mathrm{cm} \mathrm{s}^{-1}\right.$, blue and red contour lines for negative and positive values with $10 \mathrm{~cm} \mathrm{~s}^{-1}$ increment; minima and maxima are -0.86 and $1.05 \mathrm{~m} \mathrm{~s}^{-1}$, respectively), (b) IFS temperature perturbation $T^{\prime \prime}$ (K, blue and red contour lines in $1-\mathrm{K}$ increments; minima and maxima are -13.7 and $11.9 \mathrm{~K}$, respectively), and (c) NAGVEM HYPRID425 temperature perturbation $T^{\prime \prime}(\mathrm{K}$, blue and red contour lines in 1-K increments; minima and maxima are -8.9 and $7.0 \mathrm{~K}$, respectively) as function of UTC time since midnight of 18 Jul 2014 during RF25. The thin black lines are isentropes in a logarithmic spacing.

with temperature amplitudes larger than $1 \mathrm{~K}$ or vertical velocities larger than $0.1 \mathrm{~m} \mathrm{~s}^{-1}$ in the IFS. In contrast to the IFS, the high-top NAVGEM model places the wave-induced temperature perturbations at larger altitudes, Fig. 7c. The $T^{\prime \prime}$ amplitudes of the NAVGEM data are about $4 \mathrm{~K}$ smaller, that can most probably be explained by the lower spatial resolution compared to IFS. Furthermore, NAVGEM does not contain the wave structure below about 40$\mathrm{km}$ altitude.

Before investigating the origin of stratospheric waves, we present airborne Rayleigh lidar measurements from RF25 that support the reality of numerically generated gravity waves and confirm AIRS measurements as presented by Eckermann et al. (2019). Additionally, the next sections overview the spatial appearance and temporal evolution of the stratospheric gravity waves based on ERA5 data.

\section{Stratospheric gravity waves}

\section{a. Airborne lidar observations}

The airborne Rayleigh lidar measurements provide temperature observations from about $24-$ to $60-\mathrm{km}$ altitude. Figures $8 \mathrm{a}$ and $8 \mathrm{~b}$ show the temperature field overlaid by the HRES IFS temperatures. The observations near and above the stratopause appear quite noisy due to the small signal-to-noise ratio and the resulting large random errors (up to $\pm 10 \mathrm{~K}$ ) at this altitude. Nevertheless, the rather smooth IFS temperatures agree fairly well with the observed temperature distribution. Moreover, the observations as well as the IFS temperatures reveal periodic oscillations in proximity to the southernmost WP3. Alternating positive and negative $T^{\prime \prime}$ values delineate phases of gravity waves as shown in Figures $8 \mathrm{c}$ and $8 \mathrm{~d}$. Inspecting the horizontal wind-superimposed in Figs. $8 \mathrm{c}, \mathrm{d}$ reveals two interesting findings. First, the observed phase lines are tilted considerably toward the south in the negative shear zone of the PNJ above 50-km altitude. Second, the observed phase lines are nearly perpendicular within the core of the PNJ, while they are again inclined in the shear layer below the PNJ for $z<35 \mathrm{~km}$. Toward the northern edge of the PNJ, the magnitude of the observed temperature perturbations fades markedly both along the outbound and the inbound legs due to the decreasing wind.

Figure 9 juxtaposes the HRES IFS and NAVGEM HYBRID425 results with the airborne Rayleigh lidar observations along the out- and inbound legs. As in Fig. 8, the same temporal filter $\langle\cdots\rangle$ is applied to the modeled temperature profiles, see Figs. $7 \mathrm{~b}$ and $7 \mathrm{c}$. The numerically simulated patterns resemble the observations, yet, they are more coherent and smoother. Although the respective $T^{\prime \prime}$ amplitudes differ considerably, the similar shape of the spatial structures confirm that the NWP models reproduce the observed gravity waves rather well, at least partially. As stated above, the IFS $T^{\prime \prime}$ amplitude decrease rapidly above the stratopause due to the imposed divergence damping in the sponge layer starting at about $1 \mathrm{hPa}$ or 42-km altitude (e.g., Ehard et al. 2018; Polichtchouk et al. 2017, 2021). In this altitude region, NAVGEM HYBRID425 generates $T^{\prime \prime}$ amplitude larger than $1 \mathrm{~K}$ whose phase lines correspond to the airborne observations. Consistently, and according to Fig. 3 of Eckermann et al. (2018), the sponge in NAVGEM starts at higher levels of about $100 \mathrm{~km}$ allowing for larger temperature perturbation at these altitudes.

To present a quantitative comparison, we calculated the specific gravity wave potential energy (per unit mass):

$$
E_{P}=\frac{1}{2} \frac{g^{2}}{N^{2}} \frac{\overline{T^{\prime \prime 2}}}{\overline{T^{2}}},
$$

where $N^{2}=(g / T)\left(d T / d z+g / c_{p}\right)$, the overbar symbolizes a vertical average. Figure 10 depicts the latitudinal dependency of $E_{p}$ for the airborne lidar and for the model data binned into two altitude ranges below $(28-44 \mathrm{~km})$ and across the stratopause (44-60 km). Maxima of observed $E_{p}$ exist in a band from $58^{\circ}$ to about $61^{\circ} \mathrm{S}$ and the $E_{p}$ is decreasing gradually northward. This is in agreement with the subjective finding 

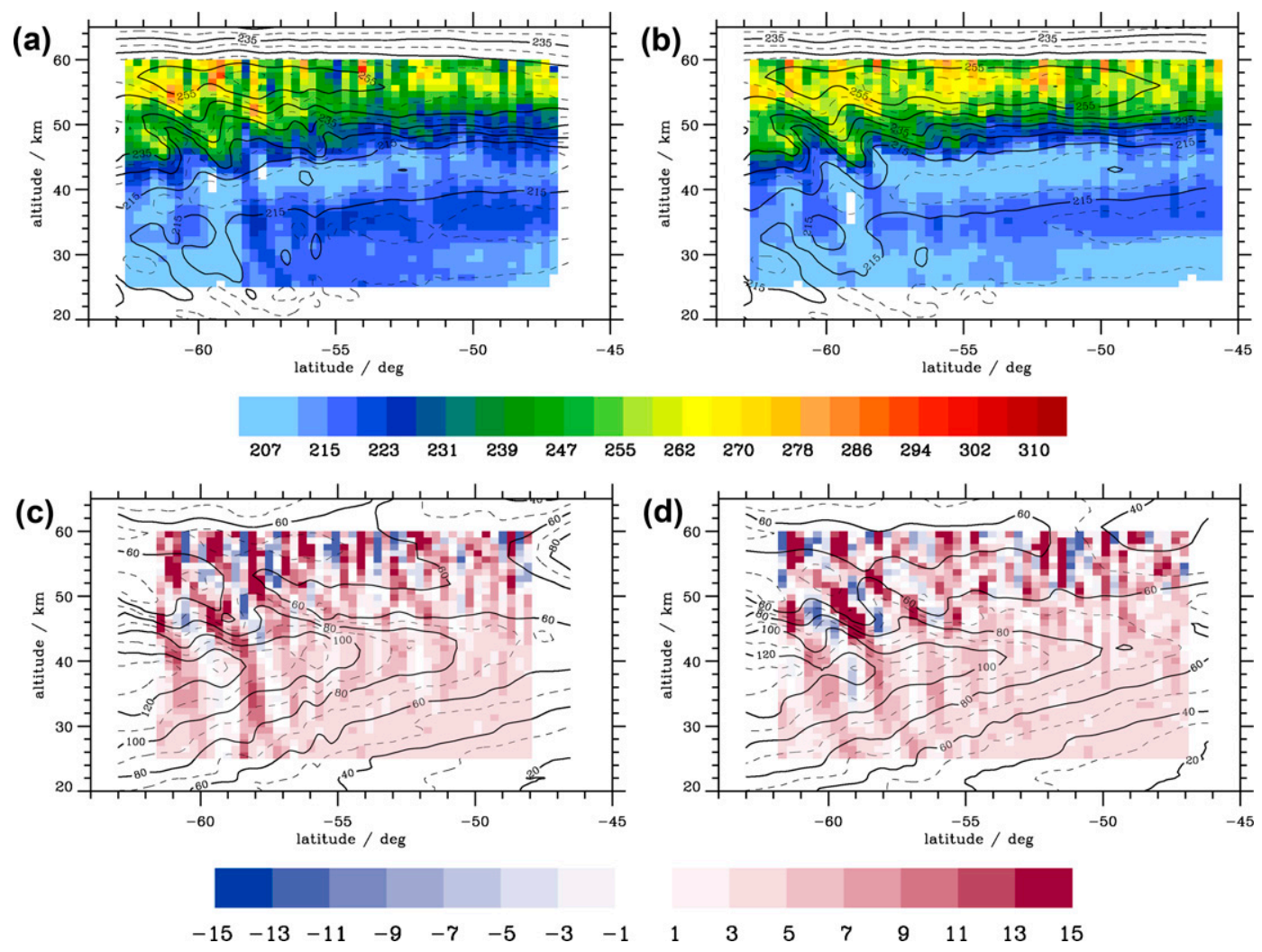

FIG. 8. (a),(b) GV Rayleigh lidar temperature (K, color coded) and IFS temperature (K, black contour lines) and (c),(d) temperature perturbations $T^{\prime \prime}$ from the Rayleigh lidar (K, color coded) and IFS horizontal wind ( $\mathrm{m} \mathrm{s}^{-1}$, black contour lines) for the (a),(c) outbound and (b),(d) inbound legs of RF25. Data: 180-s mean values of the Rayleigh lidar observations and HRES IFS.

that the observed temperature fluctuations $T^{\prime \prime}$ are larger near the southernmost way point WP3. Also, the larger values of $E_{p}$ in the upper altitude bin confirm the visual impression from Figs. $8 \mathrm{c}$ and $8 \mathrm{~d}$. The $E_{p}$ calculated with IFS data reproduce both the observed latitudinal as well as the altitude dependence; however, with a much smaller amplitude as expected by the lower $T^{\prime \prime}$ values. In contrast to the observations, the IFS shows maxima in both altitude bins at around $55^{\circ} \mathrm{S}$ along the outbound leg. A similar local maximum is also modeled by the NAVGEM HYBRID425 run. In this run, the observed maximum of $E_{p}$ at higher latitudes is not reproduced due to the small temperature perturbations in this altitude region, compare Fig. 7c to Fig. 7b. Yet, NAVGEM HYBRID425 also indicates the gradual northward decrease of $E_{p}$.

Altogether, the airborne Rayleigh lidar observations confirm the realism of the modeled wave-induced temperature perturbations, especially for the higher resolved HRES IFS data. The quantitative comparison reveals an underestimation of the simulated temperature fluctuations, yet the location and the shape seem to be in fair agreement with the observations.

\section{b. Representation in ERA5}

Figure 11 depicts the horizontal distribution of the gravity wave-induced temperature perturbations $T_{\text {ERA5 }}^{\prime}$ derived from
ERA5 data (see section 2) at four upper-stratospheric pressure levels from 30 to $1 \mathrm{hPa}$. The gravity waves overlay the poleward side of the stratospheric anticyclone and their phase lines are directed into the PNJ. Their spatial appearance and orientation was verified by AIRS observations, see Fig. 18f in Eckermann et al. (2019). As shown by the four panels in Fig. 11, their $T_{\text {ERA5 }}^{\prime}$ - amplitudes increase with increasing altitude. The gravity waves that were observed along the RF25 flight track belonged to wave packets that were aligned in NW-SE direction at all pressure levels and extended from about $130^{\circ} \mathrm{E}$ to more than $180^{\circ}$. Their oblique orientation suggests a focusing of stratospheric gravity waves originating from lower latitudes into the PNJ centered at around $60^{\circ} \mathrm{S}$.

Figures 12 and 13 complete the graphical representation of the stratospheric gravity waves by displaying longitudealtitude sections at $55^{\circ}$ and $60^{\circ} \mathrm{S}$ and latitude-altitude sections at $140^{\circ}$ and $160^{\circ} \mathrm{E}$, respectively. Whereas Fig. $12 \mathrm{a}$ only depicts one single dominating wave packet at about $160^{\circ} \mathrm{E}$ inside the core of the PNJ, the section farther south at $60^{\circ} \mathrm{S}$ shows at least three horizontally separated wave packets (identified as a vertical sequence of at least three maxima and minima in the temperature perturbations stacked obliquely on top of each other) in the region from $130^{\circ} \mathrm{E}$ to more than $180^{\circ}$. As mentioned earlier, these stratospheric waves populate the PNJ. Comparison of the south-north sections, as shown in 

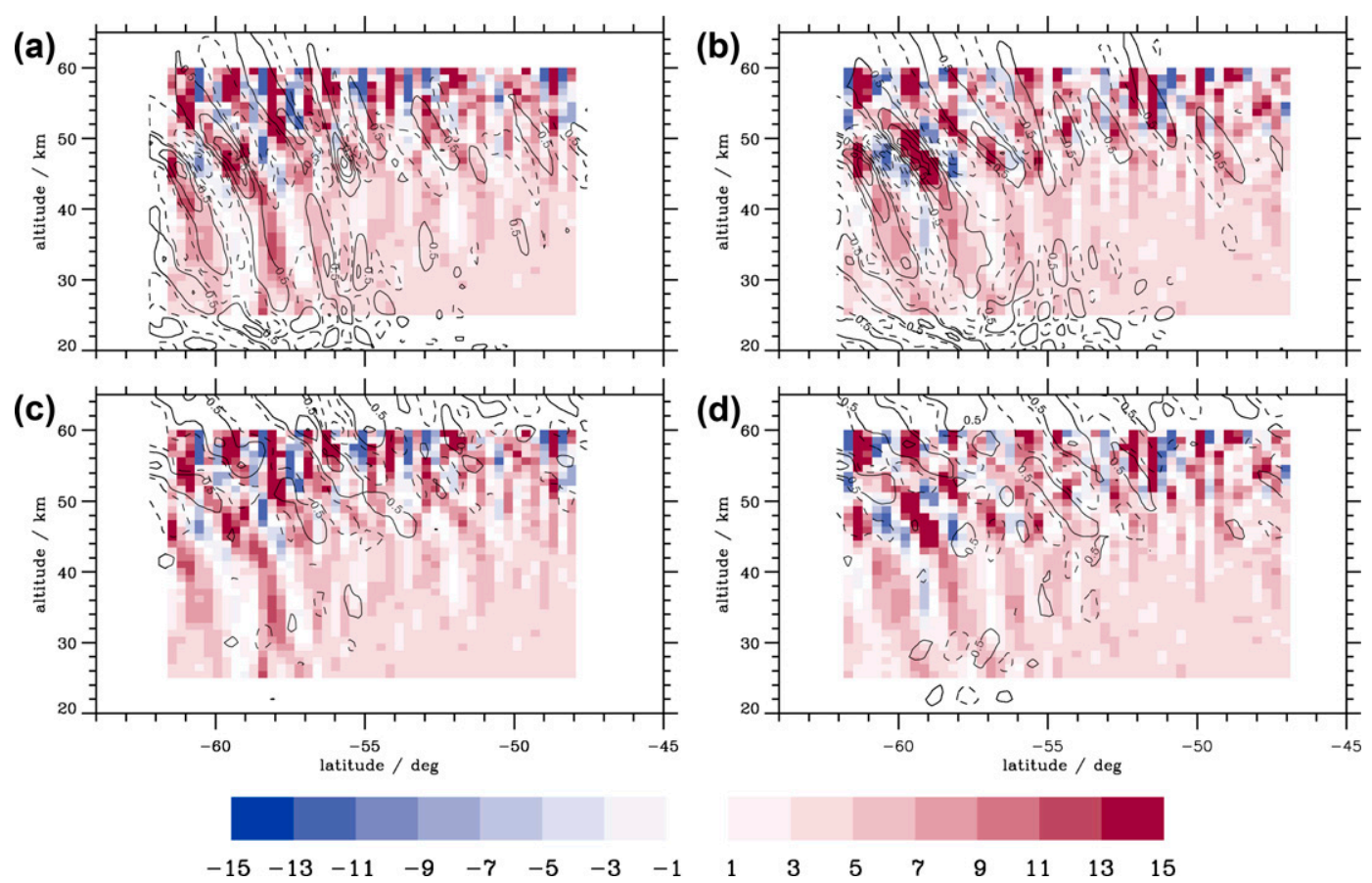

FIG. 9. GV Rayleigh lidar temperature perturbations $T^{\prime \prime}$ (K, color coded) along the (a),(c) outbound and (b),(d) inbound legs. Superimposed are temperature perturbations $T^{\prime \prime}$ (a),(b) from HRES IFS and (c),(d) from NAVGEM HYBRID425 (K, solid and dashed lines for positive and negative values). Data: 180-s mean values of the Rayleigh lidar observations and HRES IFS.

Fig. 13, indicates an increase of waves in the negative shear zone of the PNJ for the more western section. It is interesting to note, that in both figures the phase lines extend down into the lower stratosphere and appear to be connected to the polar front jet underneath.

Finally, Fig. 14 displays snapshots of the vertical component of the wave energy flux vector $\mathrm{EF}_{z}=w_{\mathrm{ERA} 5}^{\prime} p_{\mathrm{ERA} 5}^{\prime}$ (perturbations are calculated as outlined in section 2) at the same upper-stratospheric pressure levels as selected for the temperature perturbations in Fig. 11. In general, the wave energy flux vector determines in which direction and at which rate energy is being transported by the wave motion (Dutton 1976, his section 12.4.3). Obviously, as $\mathrm{EF}_{z}$ is predominantly positive, the stratospheric gravity waves propagate upward and $\mathrm{EF}_{z}$ increases with altitude. However, these values are mostly less than $1 \mathrm{~W} \mathrm{~m}^{-2}$, probably due to the underestimation of the IFS vertical velocity in the stratosphere. Nevertheless, the magnitude of the diagnosed $\mathrm{EF}_{z}$ values is consistent with early results from mesoscale numerical simulations of a non-orographic DEEPWAVE case (Kruse and Smith 2015, their Figs. 15-17) and may be characteristic for the weakness of the associated sources. In contrast to the small values found here, preliminary results from the SOUTHTRACGW campaign (Rapp et al. 2021) show stratospheric $E_{z}$ values associated with mountain waves over the Andes, reaching as high as $5 \mathrm{~W} \mathrm{~m}^{-2}$ in the ERA5 data. Time loops of plots like those shown in Fig. 14 reveal that the patches of positive $\mathrm{EF}_{z}$ propagate eastward and extend south into the PNJ in time.
This propagation of stratospheric gravity wave activity is illustrated by the temporal evolution of the area-averaged values of $\mathrm{EF}_{z}$ in eight control areas as sketched in Fig. 14a. In Fig. 15 the west-east succession of the areas centered at $55^{\circ}$ and $60^{\circ} \mathrm{S}$ is to be read from top to bottom whereby the northern four areas form the left column. For all upper-stratospheric pressure levels we diagnose an eastward propagation of about $17 \mathrm{~m} \mathrm{~s}^{-1}\left(\approx 40^{\circ}\right.$ in longitude in $36 \mathrm{~h}$ at about $60^{\circ} \mathrm{S}$, i.e., about $60 \mathrm{~km} \mathrm{~h}^{-1}$ ) of the gravity wave activity, i.e., the $\mathrm{EF}_{z^{-}}$ maxima wander right in time from one area to another. The lowest pressure levels plotted in Fig. 15 are 100 and $300 \mathrm{hPa}$ and the characteristic eastward shift of stratospheric wave activity is not reflected as these levels and the $\mathrm{EF}_{z}$ values are generally smaller compared to the levels above. It is the pronounced eastward propagation of the upper-stratospheric gravity wave activity with the same phase speed as that of $z_{\text {tropo }}$, see section $3 \mathrm{a}$, that has piqued our curiosity to search for its potential origins.

\section{Origins of the stratospheric waves}

In this section, potential source processes of the observed and simulated stratospheric waves are investigated. Orographic sources can be largely ruled out due to the lack of any significant nearby terrain (Fig. 1) and due to the non-stationarity of the stratospheric gravity waves as shown before. Here, we focus on the non-orographic excitation and analyze the flight level as well as ERA5 data. Moreover, the findings 

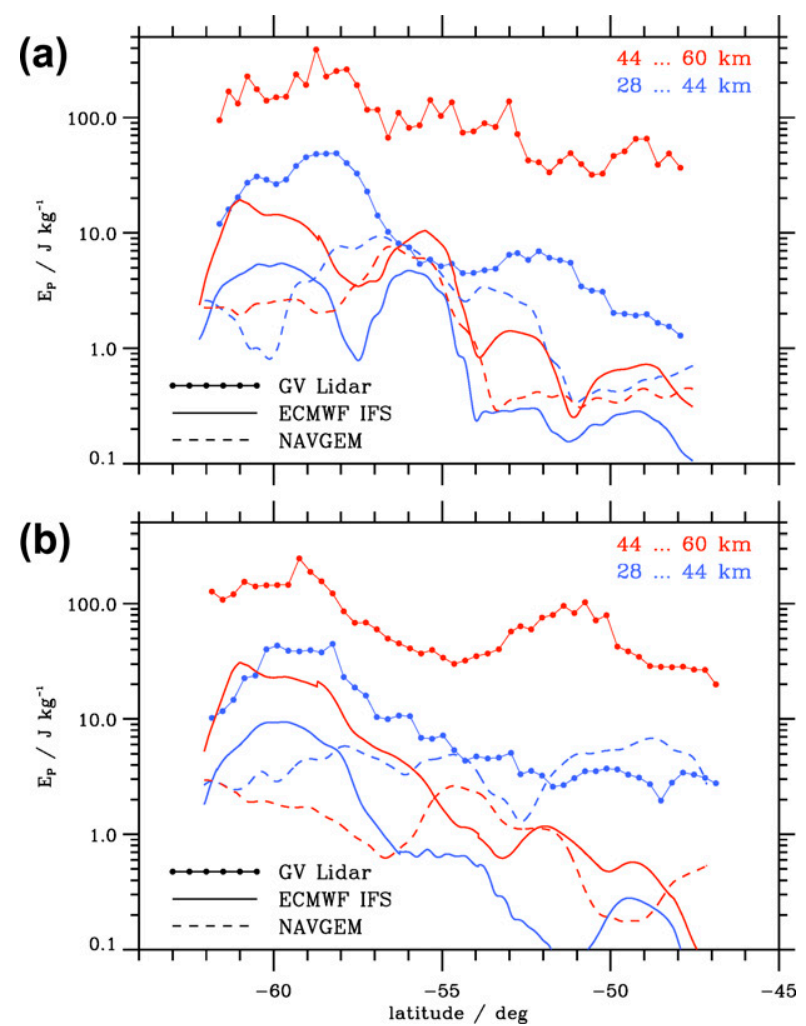

FIG. 10. (a),(b) Specific gravity wave potential energy $E_{P}$ derived from GV Rayleigh lidar temperature perturbations $T^{\prime \prime}$ (dots and solid lines) as well as derived from IFS (solid lines) and from NAVGEM HYBRID425 (dashed lines) for the RF25 outbound and inbound legs. The red and blue colors mark the 28-44- and 44-60-km altitude bins, respectively.

of the previous section, especially, the widespread appearance of the stratospheric gravity waves and their southeast propagation strongly indicate a remote source and that processes in the proximity of the RF25 flight track alone might not be the reasons for their existence. Nonetheless, we begin by examining the vertical momentum and energy fluxes from the in situ data of RF25 to quantify the waves at the flight level. This analysis should clarify whether a local source in the lower troposphere can be detected and quantified, as is usually done in mountain wave flights (Smith et al. 2008, 2016).

\section{a. Eliassen-Palm relation}

The Eliassen-Palm relation provides a functional dependence of the vertical flux of wave energy $\mathrm{EF}_{z}=w^{\prime} p^{\prime}$ and the negative scalar product of the vectors of the horizontal wind $\mathbf{U}$ and the specific momentum flux $\mathbf{M F}=\left(\mathrm{MF}_{x}, \mathrm{MF}_{y}\right)=$ $\left(u^{\prime} w^{\prime}, v^{\prime} w^{\prime}\right)$. According to Eliassen and Palm (1960), both quantities should be related in an one-to-one dependence for linearly propagating, steady, non-dissipative internal gravity waves. From the flight level in situ observations (see Fig. A3 in the appendix), the wave and momentum fluxes are calculated as follows: the out- and inbound legs were divided into 12 intervals separated by the 11 dropsonde release positions, see Fig. A3. As their average length is about $330 \mathrm{~km}$, we split these intervals in even shorter intervals by dividing their lengths by 2 and 3, respectively. For each of these intervals, all observed quantities are de-trended (linearly) and de-meaned to compute perturbation variables $\Psi^{\prime}=$ $\left(u^{\prime}, v^{\prime}, w^{\prime}, p_{c}^{\prime}\right)$. Before de-trending and de-meaning, the static pressure $p$ has to be corrected to a constant reference altitude $z_{\text {ref }}$ assuming hydrostatic balance $p_{c}(x)=p(x)+\bar{\rho} g\left(z-z_{\text {ref }}\right)$, where $z$ is the aircraft geometric altitude determined from the global positioning system (GPS) and $\bar{\rho}$ the leg-averaged density, Smith et al. (2008, 2016). From these variables, the energy and momentum fluxes are calculated and averaged over the respective interval lengths, i.e., there are 12, 24 , and 36 individual $\mathrm{EF}_{z}$ and $\mathbf{M F}$ values plotted with red, black, and blue symbols, respectively, in the scatter diagram of Fig. 16a.

Compared to previous observations during T-REX and DEEPWAVE (Smith et al. 2008, 2016), the wave energy fluxes are small as $\mathrm{EF}_{z}$ or $-\mathbf{U} \cdot \mathbf{M F}$ do not exceed $2 \mathrm{~W} \mathrm{~m}^{-2}$. Furthermore, the correlation coefficients for all considered legs are smaller than 0.6 compared to 0.86 reported by Smith et al. (2008) and 0.91 as reported by Smith et al. (2016). Second, the small flux values are distributed randomly around zero and the missing linear relation between $\mathrm{EF}_{z}$ and $-\mathbf{U}$. MF indicates that linearly propagating internal gravity waves carrying wave energy upward are not detectable straightforwardly in the data. As the in situ flight-level measurements were conducted in the lowermost stratosphere, an underlying tropospheric wave source cannot be satisfactorily detected.

Our results are not sensitive to the selection of the individual leg intervals. Figure $16 \mathrm{~b}$ show the results for the leg division used by Kruse et al. (2016). These values were not included in Fig. 5 of Smith et al. (2016). The gray symbols are the Kruse et al. (2016) results, the other symbols mark our own calculations. Compared to Kruse et al. (2016), our own de-trending and de-meaning results in slightly larger correlation coefficients, yet, the magnitude of the wave energy fluxes is rather similar. Still missing is a distinct assignment of positive $\mathrm{EF}_{z}$ and $-\mathbf{U} \cdot \mathbf{M F}$ values, which would indicate vertically propagating gravity waves. This lack of linear relationship and the observed small $\mathrm{EF}_{z}$ values in the lowermost stratosphere above the core of the polar front jet indicate that tropospheric sources of internal gravity waves might be unlikely or, at least, are not detectable in the flight-level in situ data.

\section{b. Gravity wave emission by propagating Rossby wave trains}

In this subsection, we present Hovmöller diagrams that reveal a close correlation between the analyzed stratospheric wave activity and the Rossby wave trains propagating along the midlatitude waveguide. The link to the stratospheric waves is established by Fig. 17 showing the tropopause heights and the stratospheric temperature perturbations at 0900 UTC 18 July 2014 during RF25 (Fig. 17b) and $18 \mathrm{~h}$ before (Fig. 17a) along $55^{\circ} \mathrm{S}$. Vertical sections like the ones shown in Fig. 17 manifest at least two findings: vertically propagating waves are always located directly above the tropopause depression at the various times, i.e., in the reference 

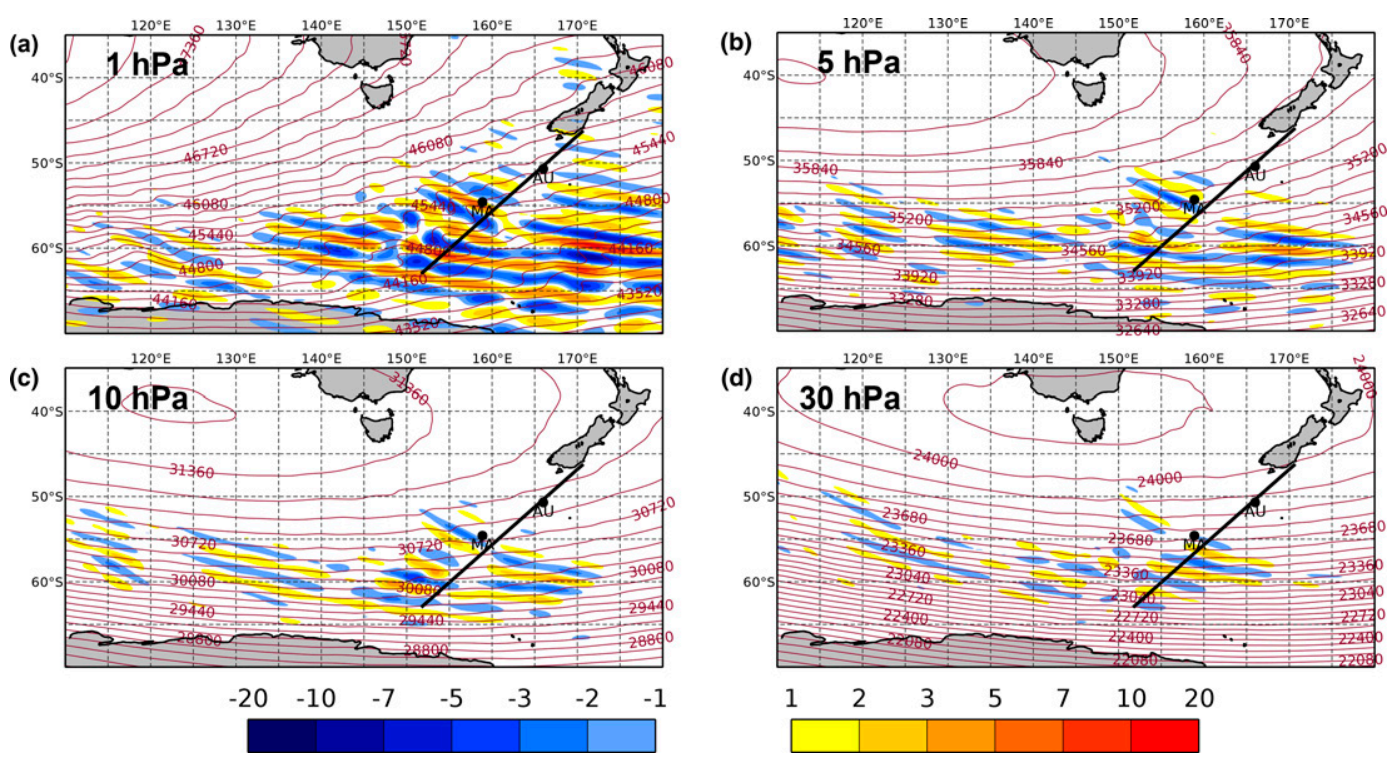

FIG. 11. Temperature perturbations $T_{\mathrm{ERA} 5}^{\prime}(\mathrm{K}$, color shaded) and geopotential height $Z$ (m, burgundy solid lines) at (a) 1 , (b) 5, (c) 10, and (d) $30 \mathrm{hPa}$ at $0900 \mathrm{UTC} 18 \mathrm{Jul} 2014$. Data: ERA5 on a $0.28125^{\circ}$ regular latitude-longitude grid.

frame of the propagating tropopause or Rossby wave train they appear as quasi-stationary phenomena. Second, Fig. 17 shows other wavelike stratospheric disturbances with smaller amplitudes. Nevertheless, the propagating tropopause depression represents a persistent but transient source of largeamplitude stratospheric gravity waves.

It is interesting to note that Prusa and Smolarkiewicz (2003) presented numerical simulations of the evolution of a gravity wave packet generated by a two-dimensional depression traveling along the tropopause. Using a realistic width of $200 \mathrm{~km}$ and an amplitude of $500 \mathrm{~m}$, a phase speed of $8.7 \mathrm{~m} \mathrm{~s}^{-1}$, and constant stratospheric wind and thermal stability they showed that a vertically propagating hydrostatic gravity wave was always located directly above the tropopause depression (Prusa and Smolarkiewicz 2003, their Figs. 1 and 2). The wave response resulted from the airflow across the moving undulation. This early numerical finding in relation to the diagnosed wave structures in the ERA5 data (Fig. 17) provokes the idea that similar processes as in the numerical simulations are at work in the real atmosphere over the Southern Ocean. Certainly, the vertical gravity wave propagation is more complicated here. First of all, the tropopause is not an impermeable surface. Nevertheless, the enhanced thermal stability of the tropopause inversion layer (Birner et al. 2002; Birner 2006; Randel et al. 2007) might mimic such an interface. Second, the presence of the PNJ tilts the gravity wave phase lines: in the negative shear above its core $(z>50 \mathrm{~km})$ the phase lines lean into the wind (west), inside the core, the phase lines are nearly vertical, and underneath $(z<30 \mathrm{~km})$, a slight tilt is detectable again in Fig. 17 similar to the results of the airborne lidar observations as discussed in section 4 a. Finally, the existence of multiple wave packets points to sources varying in space and time due to the essentially threedimensional nature of tropopause depressions and weaker potential sources along the Rossby wave train. However, compared to the values attained directly above the tropopause depression these waves have smaller amplitudes as shown in Fig. 17.

Figure 18a shows Hovmöller diagrams of the vertical energy flux $\mathrm{EF}_{z}$ at $1 \mathrm{hPa}$ and the depth of the dynamical tropopause as plotted before in Fig. 3. Maxima of $\mathrm{EF}_{z}$ lag behind the propagating tropopause depression from 0000 UTC 17 July to 1200 UTC 18 July 2014 suggesting a direct link between them. As already shown in Figs. 3b,c, this stratospheric gravity wave activity is also correlated with enhanced thermal gradients on the 2-PVU surface. This means, the appearance of enhanced stratospheric gravity wave activity is linked to the upper-level front of propagating Rossby wave train. Indeed, it is the quasi-linear propagation of the Rossby wave during this period that led to the vertical propagation of gravity waves into the stratosphere.

Figures $18 \mathrm{~b}-\mathrm{d}$ try to answer if conventional parameters (Zhang et al. 2000; Plougonven and Zhang 2014) quantifying the state of flow imbalance might provide additional possibilities of processes exciting non-orographic gravity waves. In general, the Rossby number Ro $=U /(L f)$ measures the separation of two time scales: balanced motions evolve on the advective time scale $L / U$, whereas the longest time scale for gravity wave motions is $1 / f$, with $f$ the Coriolis parameter (Pedlosky 1987). Rossby numbers are typically small for midlatitude flows. To a very good approximation, atmospheric motions at small Rossby numbers are balanced; i.e., a diagnostic relation can be established between the wind and other variables (Vallis 2017). Estimates of the Rossby number (Ro) calculated with $L=1400 \mathrm{~km}$ as approximate distance between the upper-level trough and ridge and the maximum wind speed in the jet core $U=80 \mathrm{~m} \mathrm{~s}^{-1}$ result in values of $\mathrm{Ro} \approx 0.48$. 


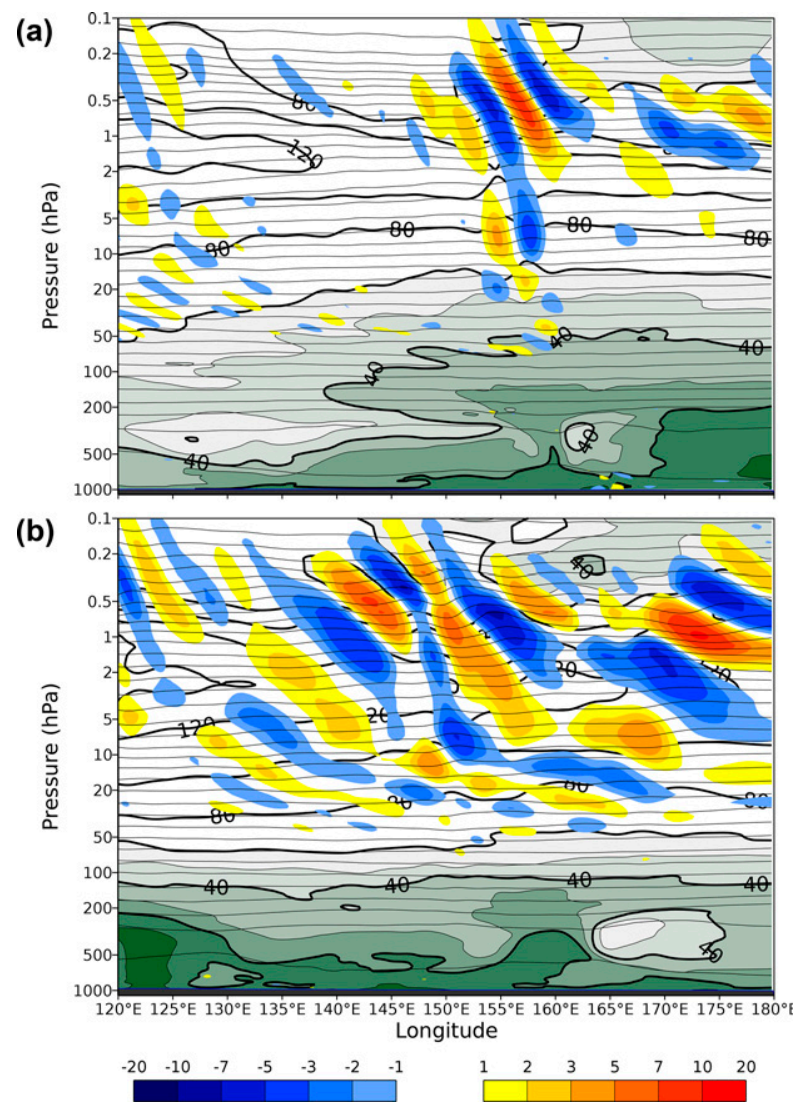

FIG. 12. Temperature perturbations $T_{\text {ERA5 }}^{\prime}(\mathrm{K}$, color shaded), horizontal wind $V_{H}\left(\mathrm{~m} \mathrm{~s}^{-1}\right.$, black lines every $20 \mathrm{~m} \mathrm{~s}^{-1}$ and green shading from 0 to $50 \mathrm{~m} \mathrm{~s}^{-1}$ in $10 \mathrm{~m} \mathrm{~s}^{-1}$ increments), and potential temperature (unlabeled logarithmic scaling, thin black lines) along (a) $55^{\circ}$ and (b) $60^{\circ} \mathrm{S}$ at $0900 \mathrm{UTC} 18 \mathrm{Jul}$ 2014. Data: ERA5. Note that lower wind speeds are shaded by darker tinting.

Figures 18b-d show the Hovmöller diagrams for three quantities at $300 \mathrm{hPa}$ : the residual of the nonlinear balance equation $|\Delta \mathrm{NBE}|$ (Zhang et al. 2000) with

$$
\Delta \mathrm{NBE}=2 J(u, v)+f \zeta-\nabla^{2} \Phi-\beta u,
$$

where $\Phi$ is the geopotential, $\beta=\partial f / \partial y$, and $J$ denotes the Jacobian, the local Rossby number $\mathrm{Ro}_{\zeta}=\zeta / f$ (the relative vorticity $\zeta=\partial v / \partial x-\partial u / \partial y$ as measure for the inverse advective time scale), and the cross-stream Lagrangian Rossby number $\mathrm{Ro}_{\perp}=\left|\mathbf{V}_{\mathrm{ag}}^{\perp}\right| / V_{H}\left(\mathbf{V}_{\mathrm{ag}}^{\perp}\right.$ is the ageostrophic wind vector perpendicular to the horizontal wind vector $\mathbf{V}$ ).

Enhanced $|\Delta \mathrm{NBE}|$ values greater than $1 \times 10^{-9} \mathrm{~s}^{-2}$ appear scattered in this plot and there seems to be no spatial correlation between the advancing Rossby wave and the $\mathrm{EF}_{z}$ pattern on the one hand side and the $|\Delta \mathrm{NBE}|$ contour lines on the other. $|\Delta \mathrm{NBE}|$ values rarely exceeds $5 \times 10^{-9} \mathrm{~s}^{-2}$ and the maximum $|\triangle \mathrm{NBE}|$ value during the considered period is less than $8 \times 10^{-9} \mathrm{~s}^{-2}$, see Fig. 18b. Zhang et al. (2001) used values larger than $10 \times 10^{-9} \mathrm{~s}^{-2}$ as indicator of strongly unbalanced flow able to emit gravity waves spontaneously.

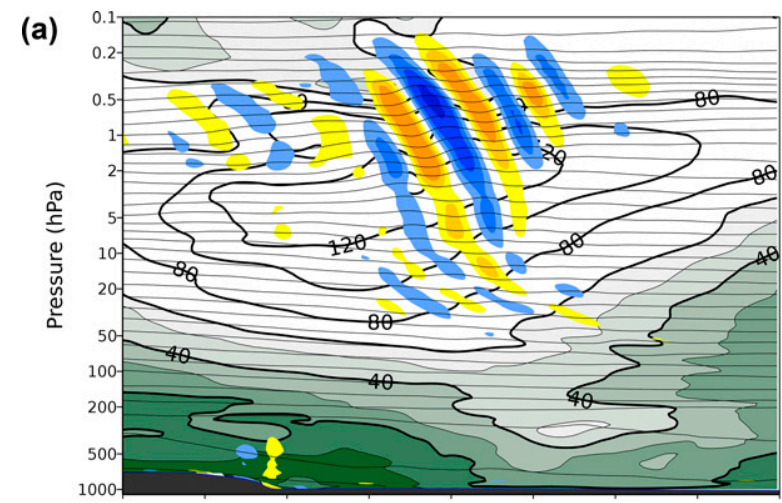

(b)

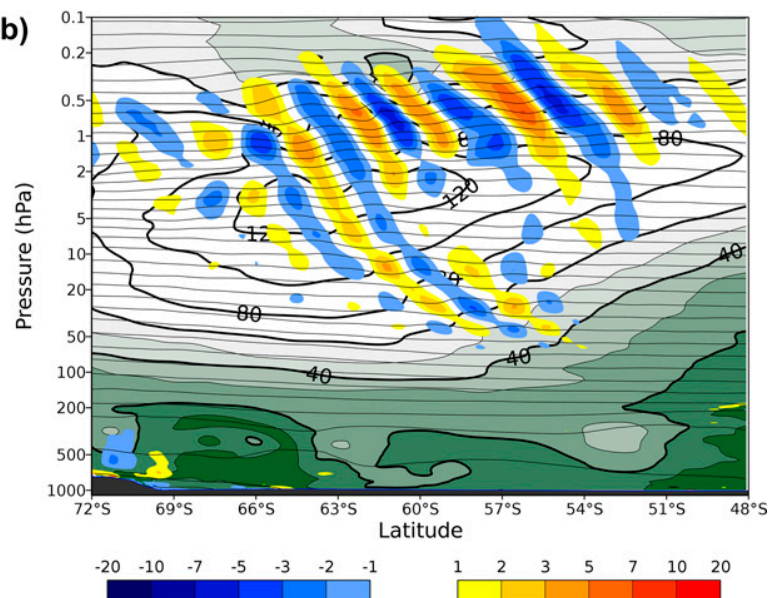

FIG. 13. Temperature perturbations $T_{\text {ERA5 }}^{\prime}$ (K, color shaded), horizontal wind $V_{H}\left(\mathrm{~m} \mathrm{~s}^{-1}\right.$, black lines every $20 \mathrm{~m} \mathrm{~s}^{-1}$ and green shading from 0 to $50 \mathrm{~m} \mathrm{~s}^{-1}$ in $10 \mathrm{~m} \mathrm{~s}^{-1}$ increments), and potential temperature (unlabeled logarithmic scaling, thin black lines) along (a) $140^{\circ}$ and (b) $160^{\circ} \mathrm{E}$ at 0900 UTC 18 Jul 2014. Data: ERA5. Note that lower wind speeds are shaded by darker tinting.

The distribution of the Rossby number $\mathrm{Ro}_{\zeta} \geq 0.4$ reveal a similar spatial pattern than the $\mathrm{EF}_{z}$ distribution, Fig. 18c. As already mentioned by Wang et al. (2009), enhanced local Rossby numbers are associated with regions inside the vortex cores and indicate strong cyclonic shear. Therefore, the spatial-temporal evolution of $\mathrm{Ro}_{\zeta}$ in Fig. 18c does reflect the passages of upper-level troughs. The cross-stream Lagrangian Rossby number $\mathrm{Ro}_{\perp}=\left|\mathbf{V}_{\mathrm{ag}}^{\perp}\right| / V_{H}$ stays smaller than 0.2, Fig. $18 \mathrm{~d}$. Tateno and Sato (2008) relate values larger 0.6 to source regions for spontaneously emitted inertia-gravity waves. In this way, these small values of $|\triangle \mathrm{NBE}|$ and $\mathrm{Ro}_{\perp}$ support the previous findings from the flight-level in situ data that gravity waves from upper-level tropospheric sources played no central role.

The spatial-temporal evolution of the magnitude of the gradient of the potential temperature $|\nabla \Theta|$ and thermal front parameter $\mathrm{TFP}_{\Theta}=-\nabla|\nabla \Theta|(\nabla \Theta /|\nabla \Theta|)$ at surfaces of constant pressure of 300 and $700 \mathrm{hPa}$ are displayed in Fig. 19. As expected, the upper-level quantities in Figs. 3a,b confirm the findings; however, the magnitude of the gradients is smaller here compared to the values at the dynamical 

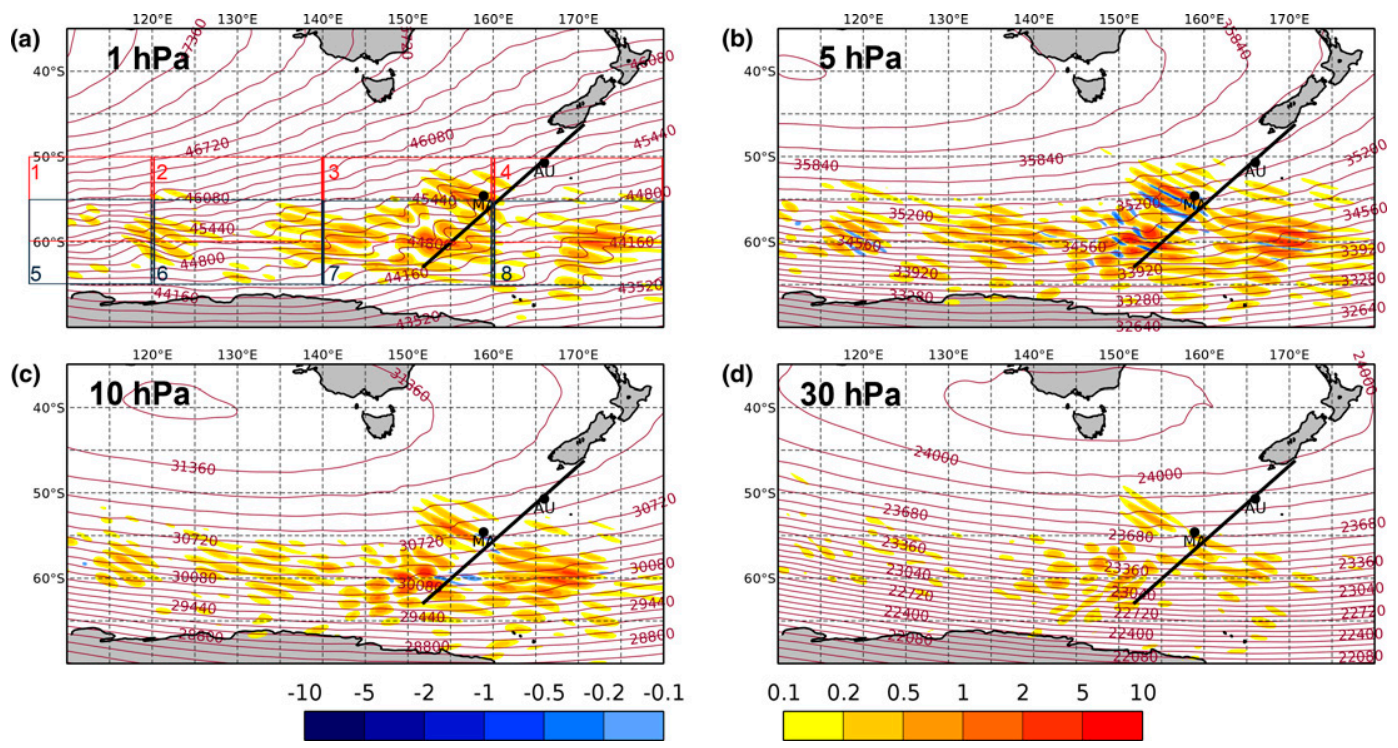

FIG. 14. Vertical energy flux $\mathrm{EF}_{z}\left(\mathrm{~W} \mathrm{~m}^{-2}\right.$, color shaded) and geopotential height $Z$ ( $\mathrm{m}$, burgundy solid lines) at (a) 1, (b) 5, (c) 10, and (d) $30 \mathrm{hPa}$ at 0900 UTC $18 \mathrm{Jul}$ 2014. In (a), the perimeters of eight control areas are drawn that are used for statistical analysis. In the meridional direction, they are $10^{\circ}$ wide and centered at $55^{\circ}$ and $60^{\circ} \mathrm{S}$, respectively. In the zonal direction, they are $20^{\circ}$ wide and are centered at $110^{\circ}, 130^{\circ}, 150^{\circ}$, and $170^{\circ} \mathrm{E}$, respectively. Data: ERA5 on a $0.28125^{\circ}$ regular latitude-longitude grid.

tropopause. The same quantities taken at $700 \mathrm{hPa}$ do not reveal a close correlation with the progressing stratospheric wave activity supporting the idea that the stratospheric gravity wave activity is strongly linked to the upper-level dynamics.

\section{c. Stratospheric gravity wave sources}

We also calculated $|\Delta \mathrm{NBE}|$ at upper-stratospheric pressure levels 1 and $5 \mathrm{hPa}$. Interestingly, the values were exceeding $100 \times 10^{-9} \mathrm{~s}^{-2}$ that would imply a potential of spontaneously emitted gravity waves from the PNJ. However, these values were calculated using the full spectral resolution of the ERA5 data. Inspecting the different terms in Eq. (3), we found that the Laplacian dominates the $|\triangle \mathrm{NBE}|$ values at these pressure levels. As shown in Fig. 4a, the geopotential height field is strongly undulated and, eventually, the second derivative becomes large. Therefore, we retrieved spectrally truncated fields at total horizontal wavenumbers 21 and 106 and calculated $|\Delta \mathrm{NBE}|$. The $|\Delta \mathrm{NBE}|$ magnitude was drastically reduced and never exceeded $10 \times 10^{-9} \mathrm{~s}^{-2}$. So, we conclude that spontaneously emitted gravity waves from the PNJ cannot explain the observed and analyzed stratospheric gravity waves.

\section{Conclusions}

The DEEPWAVE research flight RF25 was one of the very few airborne expeditions to study both the tropospheric as well as stratospheric flow through a unique combination of multiple in situ and remote sensing instruments. The analysis of the Rayleigh lidar temperature measurements documented stratospheric gravity waves near $60^{\circ} \mathrm{S}$ with phase lines varying with altitude due to the presence of a strong polar night jet. The combination of the observations and high-resolution NWP model outputs allowed a thorough interpretation of this case.

The results of our investigation provide evidence that stratospheric gravity wave patterns (such as those detected at RF25) can be excited by eastward-migrating Rossby wave trains along the midlatitude waveguide. The basic kinematic process is the relative stratospheric airflow past a propagating tropopause depression associated with the upper-level front of the Rossby waves. During the Rossby wave life cycle, there are periods of quasi-linear phase progression that can persistently excite gravity waves. Under favorable wind conditions, these gravity waves propagate vertically into the stratosphere. Depending on the ambient flow and the latitude of the PNJ, the stratospheric gravity waves can be refracted into the PNJ. Knowledge of the combination of both processes could help to explain the observed and modeled widespread occurrence of stratospheric gravity waves over the Southern Ocean far from major topographic barriers. In the light of the current case study, the modeled strong deceleration of the PNJ (see Fig. 4) might be a result of the enhanced gravity wave drag acting during the period when stratospheric gravity waves were excited by the progressive Rossby wave train. Furthermore, the identified mechanism might also contribute to the observed enhanced stratospheric wave energy in the Southern Hemispheric gravity wave belt. In this way, our findings are in agreement with the results of Hendricks et al. (2014), who showed a correlation of the Eady baroclinic growth rate at $525 \mathrm{hPa}$ with the stratospheric wave energy in the gravity 

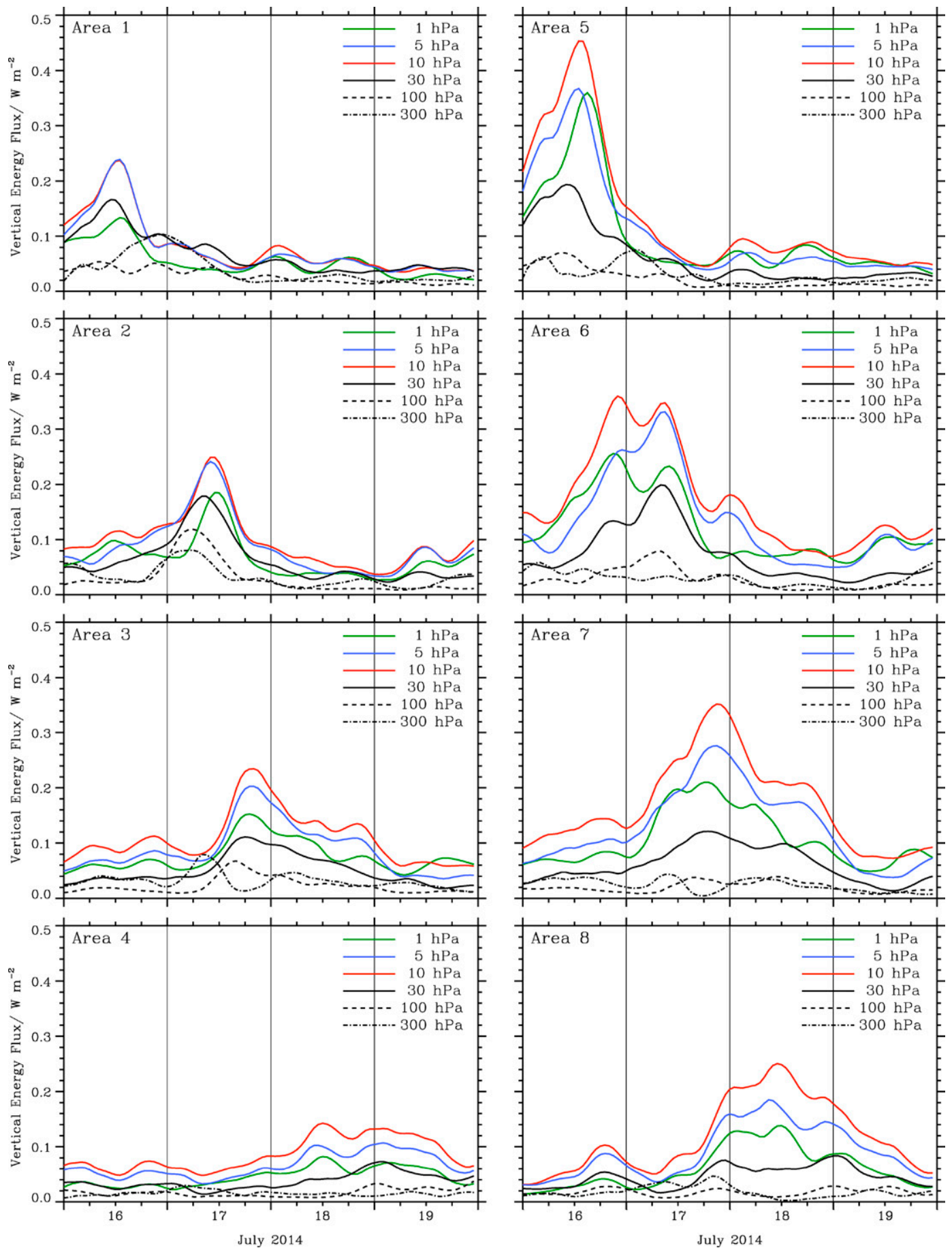

FIG. 15. Temporal evolution of the area-averaged $\mathrm{EF}_{z}$ in the different control areas: (left, top to bottom) 1-4 and (right, top to bottom) 5-8. Data: ERA5.

wave belt. Here, we identified at least one kinematic process that leads to large-amplitude stratospheric gravity waves.

As mentioned in the introduction, the proposed mechanism has similarities to the "moving mountain mechanism," which has been proposed as one source of convective gravity waves (Pfister et al. 1993). In addition, other studies exist that demonstrate that an oscillating and propagating tropopause generates stratospheric gravity waves. The classic example is the Plumb-McEwan laboratory experiment, which explains 
(a)

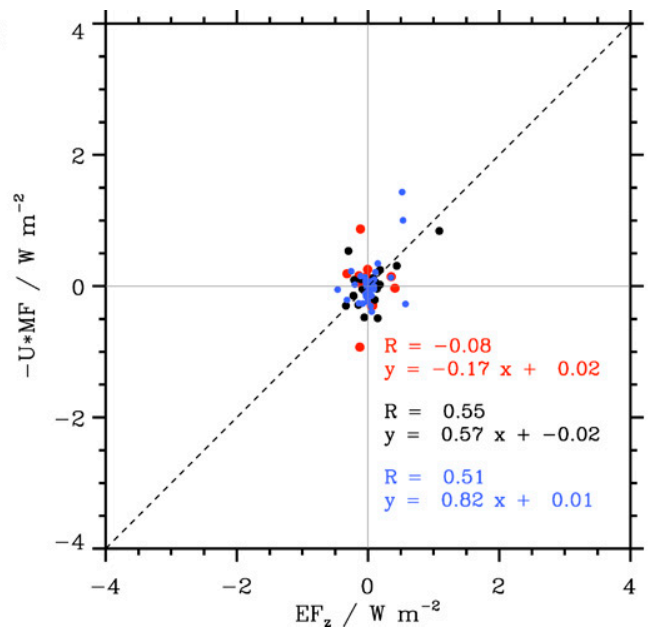

(b)

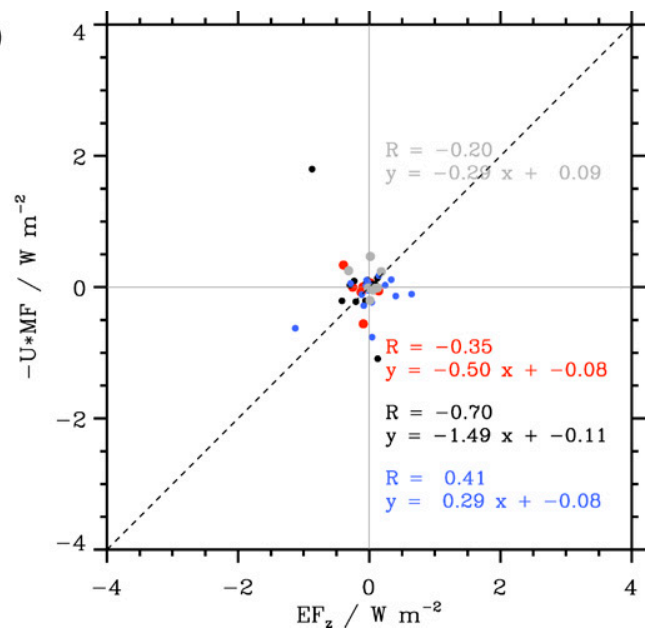

FIG. 16. (a) The scalar product of $-\mathbf{M F} \cdot \mathbf{U}$ vs $\mathrm{EF}_{z}$ for the leg division as shown in Fig. A3. The red colors mark the basis 12 legs, black symbols mark the 24 legs, and the blue symbols mark the 36 sublegs. (b) As in (a), but for the leg divisions as proposed by the Yale group whereat the gray symbols are their own provided values (Kruse et al. 2016).

the wave-mean interaction leading to the quasi-biannual oscillation (Plumb and McEwan 1978). This experiment was replicated in sophisticated numerical simulations by Wedi and Smolarkiewicz (2006). For another application, Prusa et al. (1996) designed 2D numerical experiments to test the effects of forcing by "realistic" tropospheric sources exciting a spectrum of stratospheric gravity waves. To this end, they implemented a time-varying lower surface in their numerical model. For an isothermal stratospheric under summer solstice conditions, they investigated the different
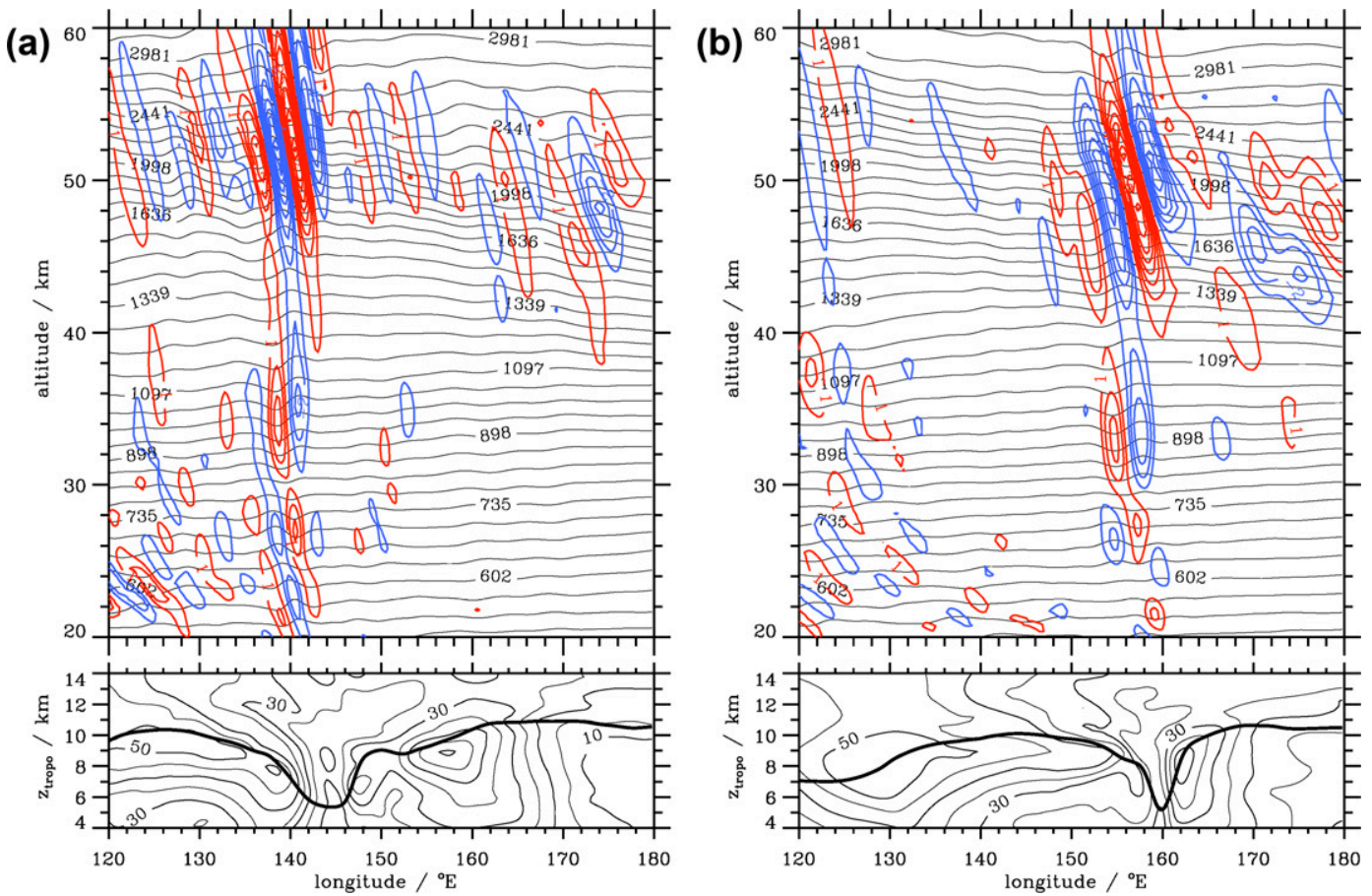

FIG. 17. (top) Temperature perturbations $T_{\mathrm{ERA} 5}^{\prime}(\mathrm{K}$, red and blue contour lines) and potential temperature (K, black lines in logarithmic scaling) along $55^{\circ} \mathrm{S}$ at (a) 1500 UTC $17 \mathrm{Jul}$ and (b) $0900 \mathrm{UTC} 18 \mathrm{Jul}$ 2014. (bottom) The height of the dynamical tropopause (thick black lines, meridional average from $52.5^{\circ}$ to $\left.57.5^{\circ} \mathrm{S}\right)$ and the horizontal wind $\left(\mathrm{m} \mathrm{s}^{-1}\right.$, thin black lines) at the same instances. Data: 1-hourly ERA5 data. 
(a)

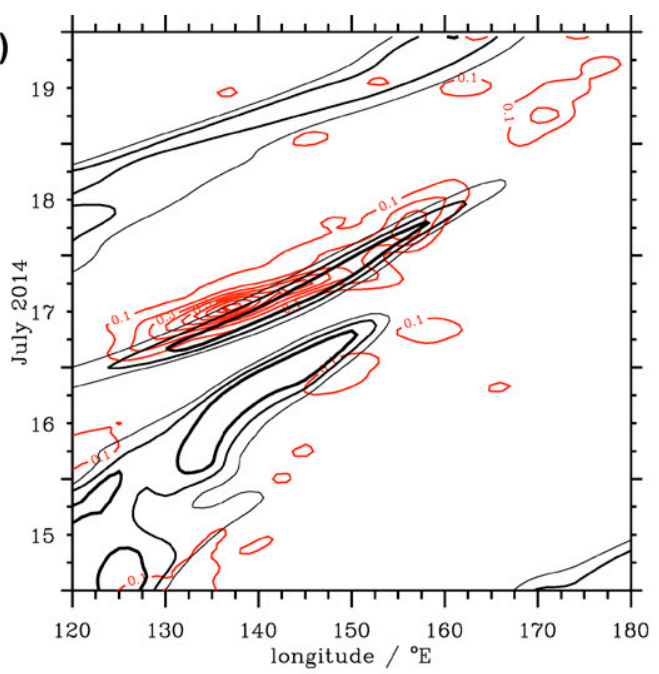

(c)

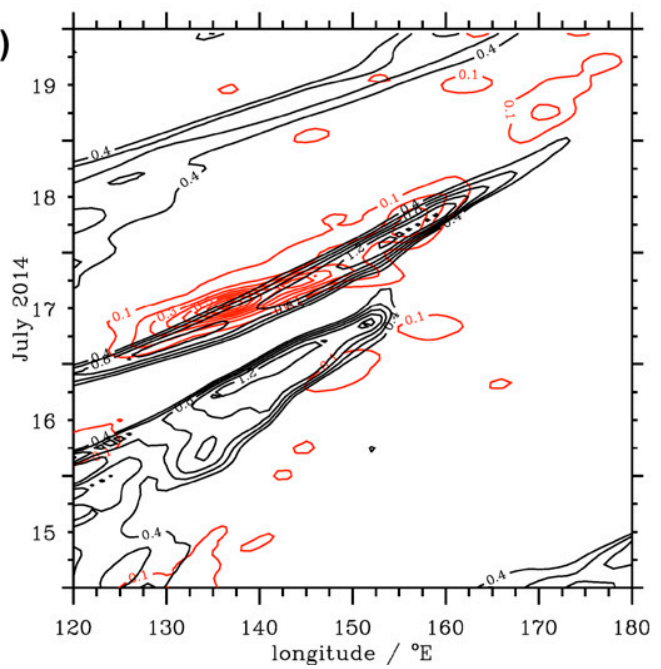

(b)

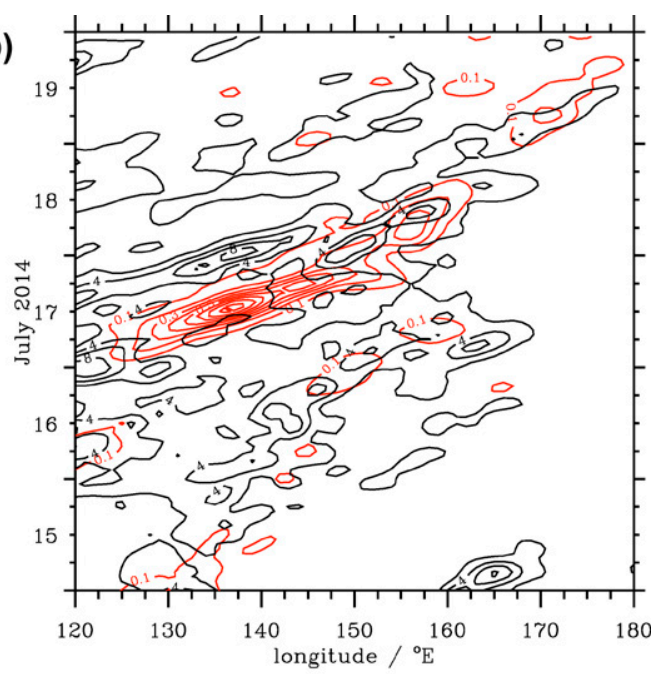

(d)

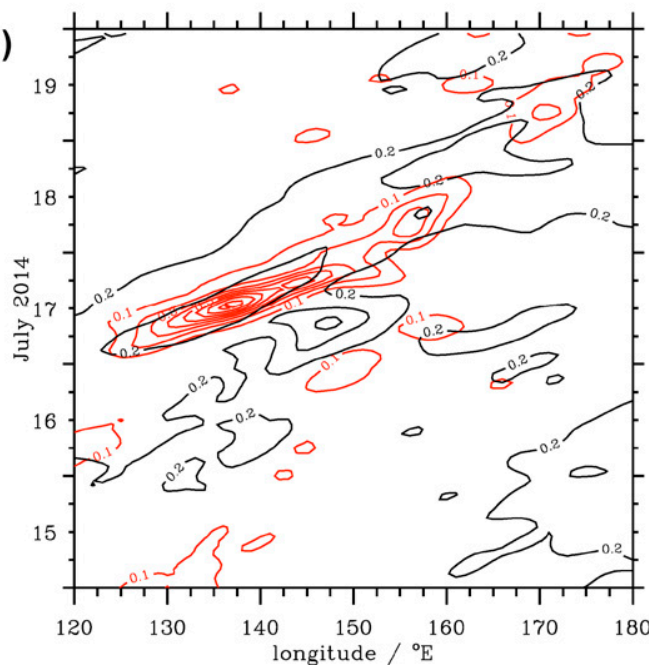

FIG. 18. Hovmöller diagrams of the vertical energy flux $\mathrm{EF}_{z}$ at $1 \mathrm{hPa}\left(\mathrm{W} \mathrm{m}^{-2}\right.$, red contour lines in all panels) overlaid with (a) the tropopause depth, (b) the residuum of the nonlinear balance equation $|\Delta \mathrm{NBE}|$ at $300 \mathrm{hPa}$, (c) the local Rossby number $\mathrm{Ro}_{\zeta}$ at $300 \mathrm{hPa}$, and (d) the cross-stream Rossby number $\mathrm{Ro}_{\perp}$ at $300 \mathrm{hPa}$ along $55^{\circ} \mathrm{S}$. Data: 1-hourly ERA5 data averaged over a latitudinal band from $52.5^{\circ}$ to $57.5^{\circ} \mathrm{S}$ and interpolated on a $1^{\circ}$ regular longitude grid.

breaking processes in the mesosphere due to the flow over tropopause undulations of different widths.

Thus, this study demonstrates the direct relationship between transient Rossby waves and stratospheric gravity waves. This relationship is based on the unique combination of observed stratospheric gravity waves and their near-realistic representation in NWP models. Furthermore, the modern NWP models offer the possibility to partially resolve gravity waves and thus a relationship between their energy flux and various tropospheric parameters could be established, which allows to infer their sources for this particular case. In the Southern Hemisphere, the excitation of stratospheric gravity waves by propagating Rossby wave trains could be a dominant mechanism in the midlatitude waveguide far from any orographic sources. Nevertheless, there are still unanswered questions and some of them that could be explored in the future are listed at the very end:

1) What determines the frequency and wavelengths of the excited stratospheric gravity waves and how does these quantities depend on the atmospheric parameters like local Rossby number, depth of the tropopause, and strength of the upper-level front?

2) Can satellite observations of stratospheric gravity waves in combination with high-resolution NWP models be used to identify more cases to substantiate the proposed mechanism?

3) Is there a statistical relation between propagating Rossby wave trains and stratospheric gravity wave activity for the austral winter months of May, June, and July? 
(a)

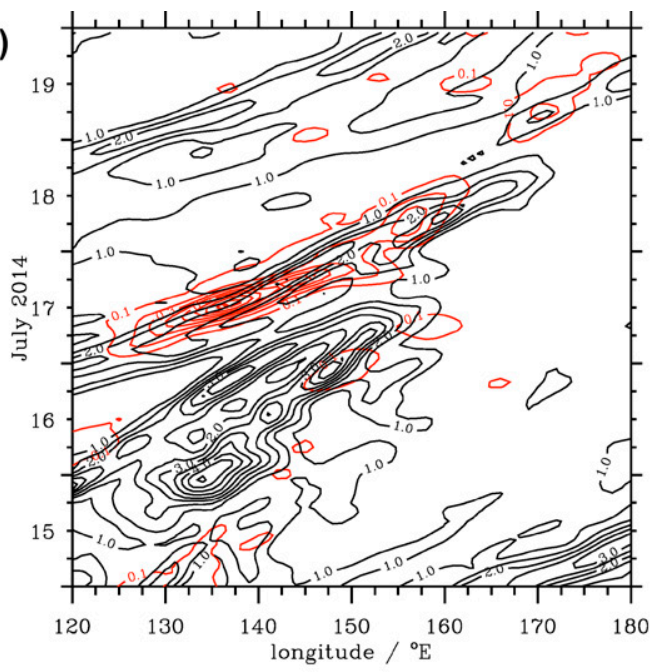

(c)

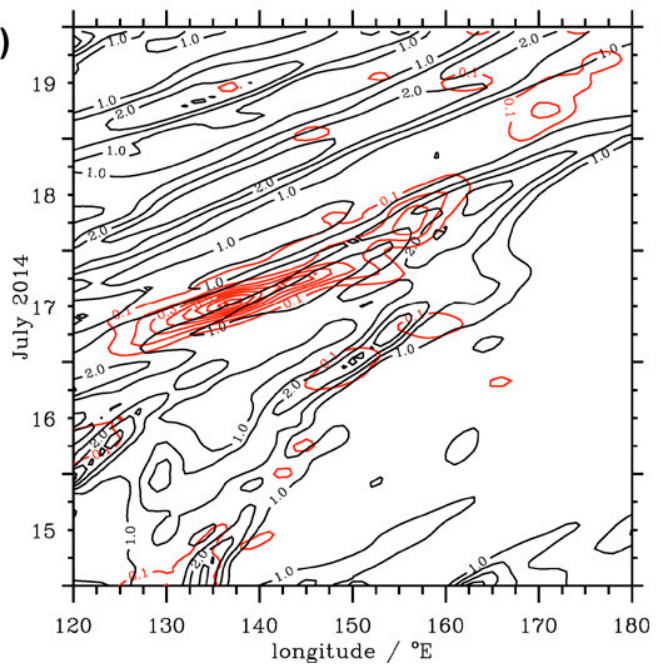

(b)

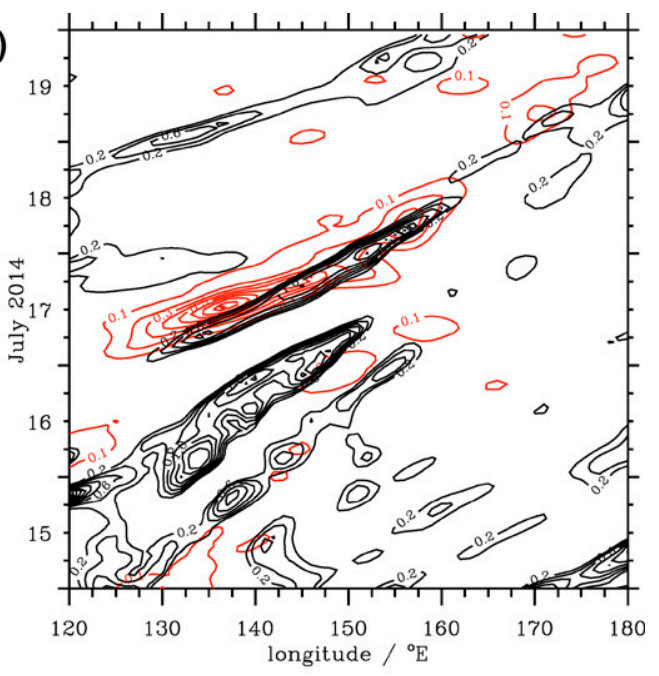

(d)

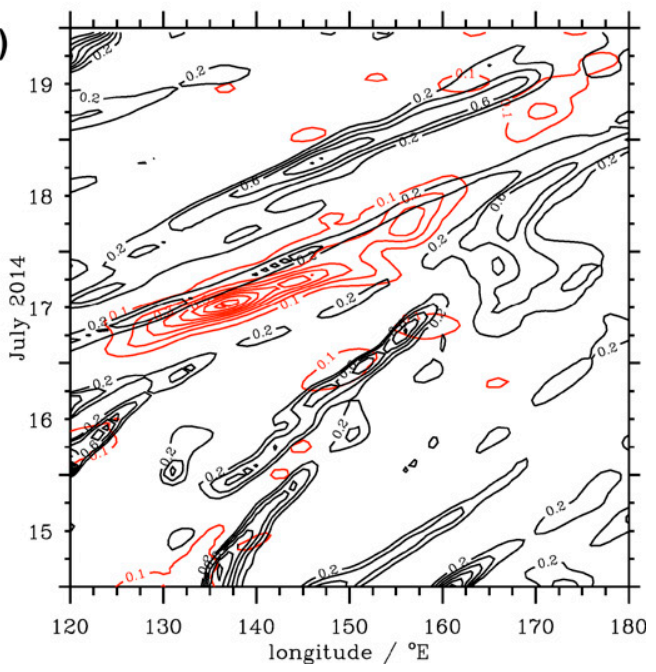

FIG. 19. Hovmöller diagrams of the vertical energy flux $\mathrm{EF}_{z}$ at $1 \mathrm{hPa}\left(\mathrm{W} \mathrm{m}^{-2}\right.$, red contour lines in all panels) overlaid with $|\nabla \Theta|$ at (a) 300 and (c) $700 \mathrm{hPa}$ and the thermal front parameter TFP ${ }_{\Theta}$ at (b) 300 and (d) $700 \mathrm{hPa}$ along $55^{\circ} \mathrm{S}$. Data: 1-hourly ERA5 data averaged over a latitudinal band from $52.5^{\circ}$ to $57.5^{\circ} \mathrm{S}$ and interpolated on a $1^{\circ}$ regular longitude grid.

4) Is there a simple possibility to parameterize the identified processes in global circulation models used for climate studies which usually lack the spatial resolution to resolve tropopause depressions properly?

Acknowledgments. This research was partly funded by the Deutsche Forschungsgemeinschaft (DFG) via the Project MSG Waves (GW-TP/DO 1020/9-1, PACOG/RA 1400/6-1) and by the German research initiative "Role of the middle atmosphere in climate" (ROMIC) under Grant 01LG1206A provided by the Federal Ministry for Education and Research. Access to ECMWF data was granted through the special project "Deep Vertical Propagation of Internal Gravity Waves" by $\mathrm{AD}$. Data were provided by NCAR/EOL under sponsor- ship of the National Science Foundation. The GV Rayleigh lidar operations and data analysis by BPW were supported by NSF Grant AGS-1338646SDE, which was supported by the Office of Naval Research through the base 6.1 and Platform Support programs (PEN 61153N).

Data availability statement. Aircraft data, radiosonde data, and the NAVGEM reanalyses interpolated on the GV flight track have been provided by NCAR/EOL under the sponsorship of the National Science Foundation (https://data.eol.ucar.edu). ECMWF'S HRES IFS operational forecast and analyses as well as the ERA5-data can be freely accessed from https://www. ecmwf.int/en/forecasts/datasets/set-i and https:/www.ecmwf.int/ en/forecasts/datasets/reanalysis-datasets/era5, respectively. 


\section{APPENDIX}

\section{Comparison with IFS Data}

\section{a. Radiosonde launches from Macquarie Island}

Vertical profiles of temperature, horizontal wind, and wind direction from seven consecutive radiosonde soundings from Macquarie Island are used to provide a validation the ECMWF IFS analyses and forecasts in the troposphere and lower stratosphere. The zonally oriented balloon trajectories as plotted in Fig. 1 reveal that the soundings released from 0000 UTC 16 July to 0000 UTC 19 July 2014 stayed west of the GV flight track. The ECMWF profiles (solid lines in Fig. A1) are interpolated on the trajectories of the balloons for the respective analysis or forecast times, the dashed lines in Fig. A1 give vertical profiles directly above Macquarie Island.

The stratospheric wind direction was predominantly west whereas the tropospheric wind turned from northerlies to westerlies during the three days. The temporal evolution of the wind and temperature profiles reflects a transition period characterized by a growing jet whose core location descended by about $2 \mathrm{~km}$. This is consistent with the tropopause subsiding in the period from 1200 UTC 17 July to 0600 UTC 18 July 2014 as seen by the temperature profiles. At this time, the tropospheric jet attained about $50 \mathrm{~m} \mathrm{~s}^{-1}$ at 8 -km altitude. Only $3 \mathrm{~h}$ later, the observed wind dropped to about $20 \mathrm{~m} \mathrm{~s}^{-1}$ at the same altitude. This finding is in agreement with the fast eastward propagation of the jet entrance region; see the $300 \mathrm{hPa}$ wind in Fig. 4d. The transition period on 18 July 2014 is also characterized by small wavelike disturbances in temperature and wind in the stratosphere. The agreement between the IFS and the radiosonde observations is quantitatively very good. In some profiles, IFS winds and temperatures even follow the observed wave structure in the stratosphere, e.g., the 1200 UTC 18 July 2014 sounding. The largest deviations in horizontal wind occur for the 0900 UTC sounding on 18 July 2014 where IFS forecasts with a lead time of $9 \mathrm{~h}$ were used. All other comparisons are close to regular analysis times, which might explain the agreement as the radiosonde data were assimilated in the IFS.

\section{b. Dropsonde and MTP profiles}

The retrieved MTP temperature data are compared to the dropsonde and IFS data. Figure A2 presents three selected vertical profiles at the dropsonde release locations D1, D3, and D6 along the outbound leg. The agreement between MTP temperatures and dropsonde observations degrades drastically for increasing distance from the flight level. At large distances from flight level, the influence of the measurements is small and the retrieved profile is largely based on the a priori radiosonde profile. Furthermore, the MTP profiles seem to be set-off vertically from the observed temperature profiles in the southern portion of the leg. As noted above, the southern portion of the leg is where the aircraft was furthest away from radiosonde profiles that would provide reasonable a priori data for first guesses. Additionally, the MTP and dropsonde observations are made on different time scales (17 s for the MTP, several
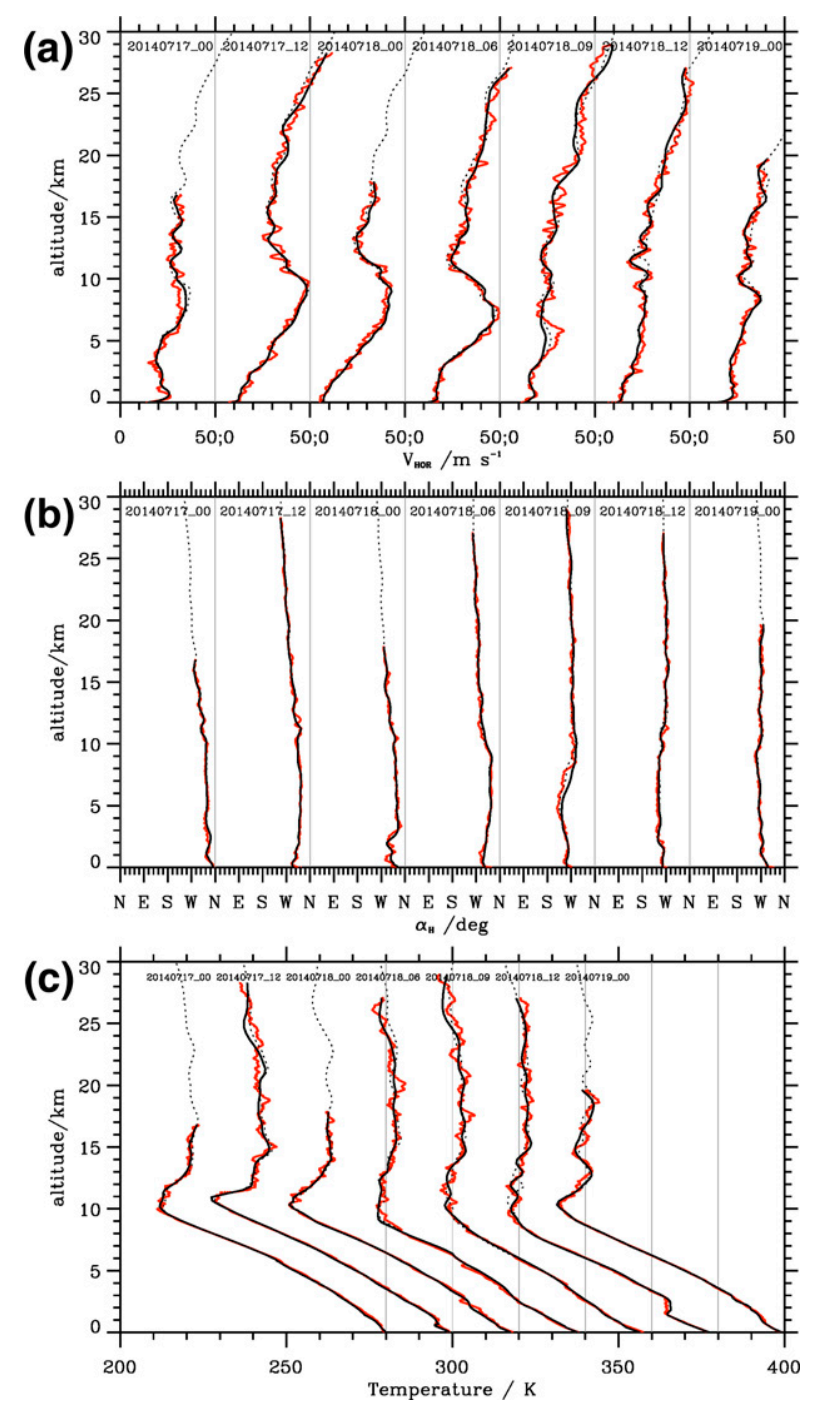

FIG. A1. Radiosonde profiles (red dots) of (a) horizontal wind, (b) wind direction, and (c) absolute temperature for seven launch times from Macquarie Island (from left to right): 2315:14 UTC $16 \mathrm{Jul}, 1115: 13$ UTC $17 \mathrm{Jul}$, 2315:06 UTC $17 \mathrm{Jul}$, 0619:59 UTC 18 Jul , 0900:24 UTC 18 Jul, 1115:30 UTC 18 Jul, and 2315:05 UTC 18 Jul 2014. Black solid lines: ECMWF interpolated profiles along the balloon trajectories. Black dashed lines: ECMWF vertical profiles above Macquarie Islands.

minutes for the dropsonde). Last but not least, the MTP looks in the direction of flight while the dropsonde moves away from the aircraft in the direction of the wind. So they may not always be observing the same portion of the atmosphere, and in cases with a high degree of spatial and temporal variation, the two profiles might be expected to show differences in the wave structure. In contrast to the detected deviations with the MTP data the IFS profiles agree rather well with the dropsonde profiles. Again, as mentioned above, this might be not surprising as the dropsonde data went into the data assimilation at the ECMWF. 

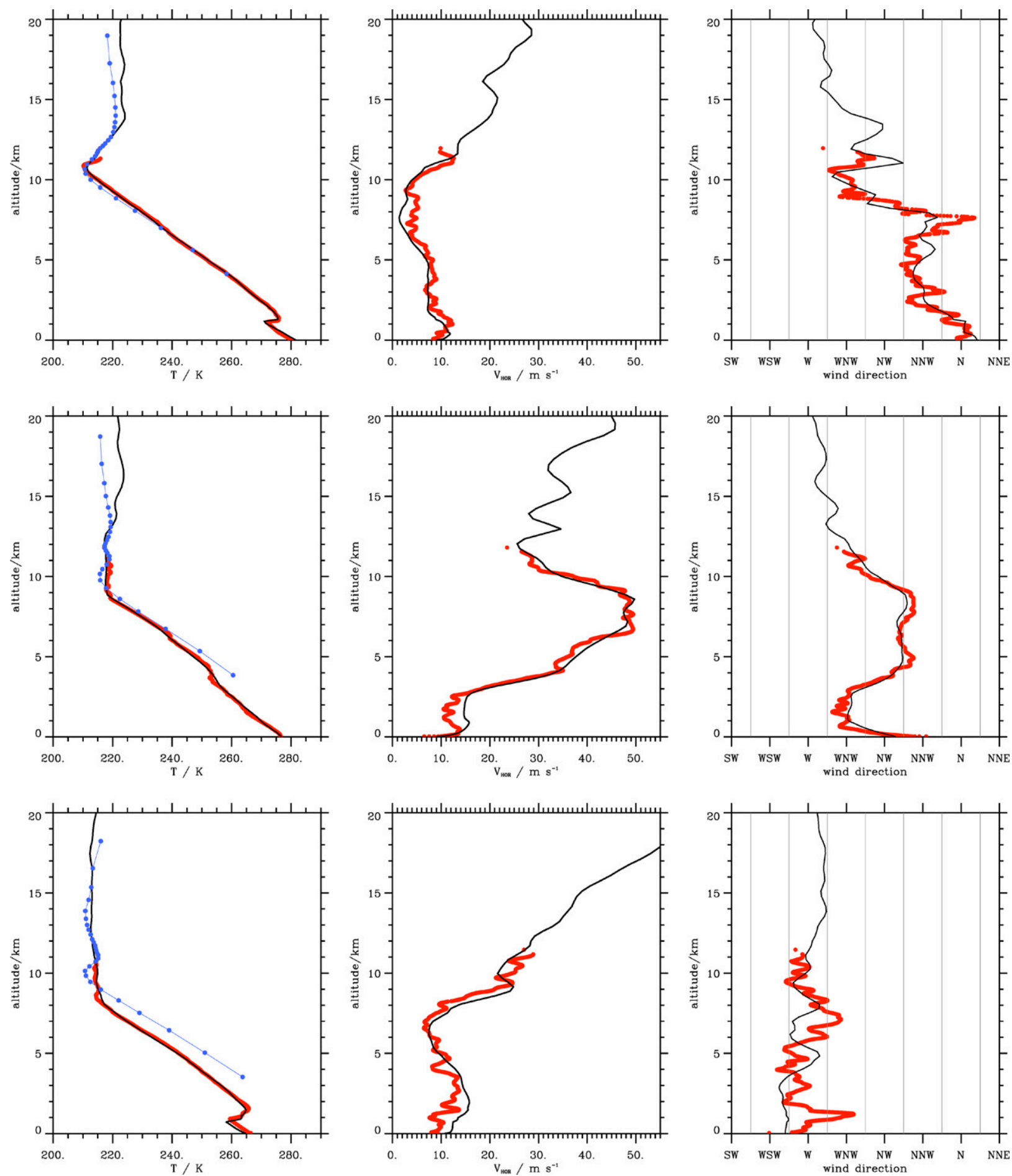

FIG. A2. (left) Absolute temperature (K), (center) horizontal wind ( $\mathrm{m} \mathrm{s}^{-1}$ ), and (right) wind direction from dropsonde positions (top) D01, (middle) D03, and (bottom) D06 as indicated in Fig. 1. Red: dropsonde observations; blue: MTP temperature near release time; black: IFS data interpolated in space and time on the dropsonde release locations. 


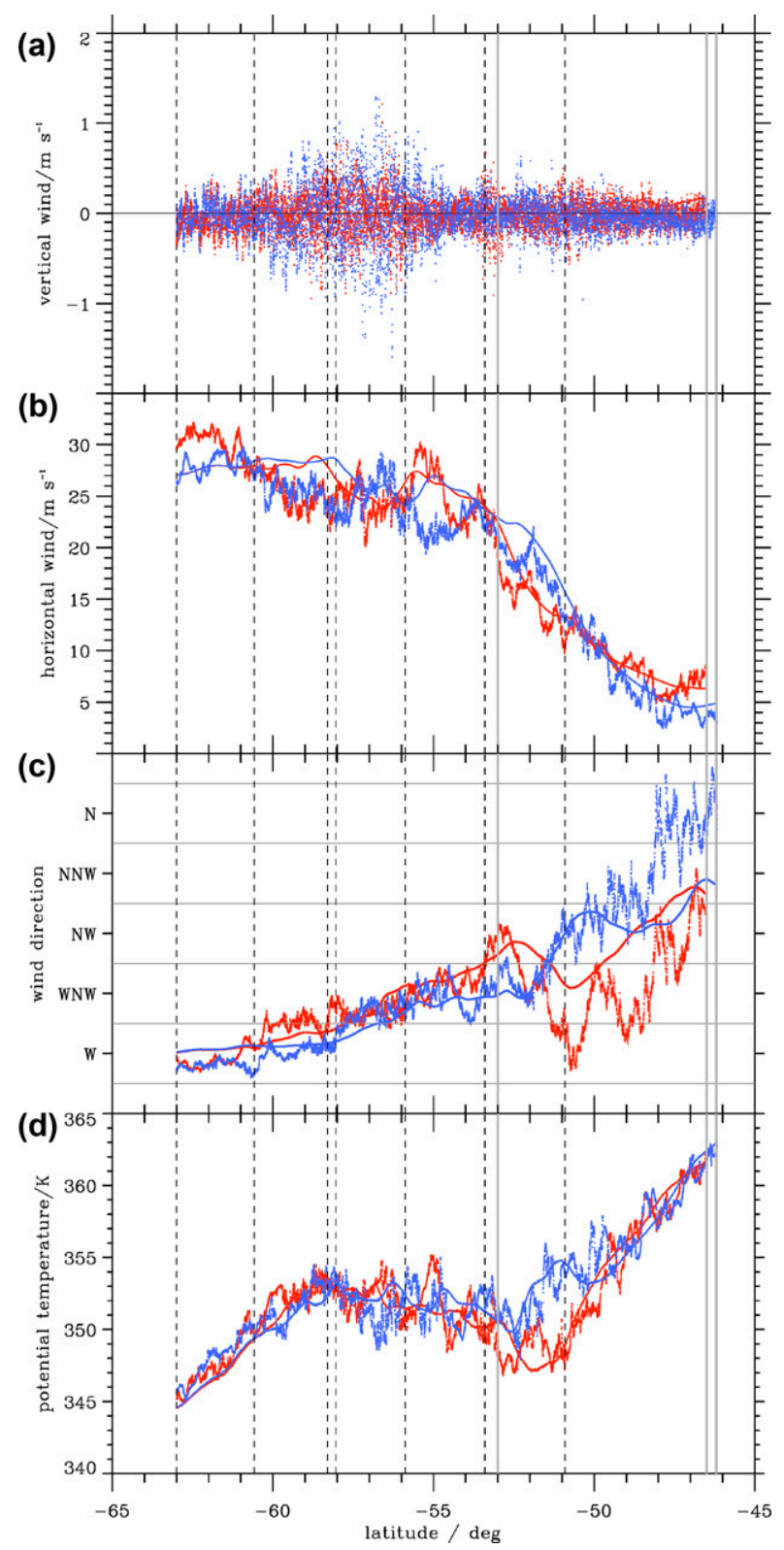

FIG. A3. (a) Vertical wind, (b) horizontal wind, (c) wind direction, and (d) potential temperature along outbound (red) and inbound (blue) legs of RF25. Dots: GV in situ flight-level data (WIC, WSC, WDC, and THETA); solid lines: IFS 6-hourly analyses and 1-hourly forecasts interpolated in space and time on the flight track. The IFS vertical winds are multiplied by a factor of 10 . The vertical dashed lines denote the positions of the dropsonde releases as shown in Fig. 1 and the vertical gray lines marks the waypoints WP1 and WP2 as listed in Table 1.

\section{c. In situ observations at FL400}

Figure A3 overviews the flight-level wind and potential temperature observations. Furthermore, the dropsonde release positions are marked with dashed vertical lines. Their locations and the northernmost way points WP1 border the 12 segments used for the analysis of the wave energy and momentum fluxes in section 5. The largest fluctuations in the vertical wind occur at around $58^{\circ} \mathrm{S}$, Fig. A3a. The magnitude of the horizontal wind increased from about 5 to $20 \mathrm{~m} \mathrm{~s}^{-1}$ from WP1 to WP2. Here, the $V_{H}$ profiles do not vary strongly between the outbound and inbound legs, Fig. A3b. However, the wind direction turned from northwesterlies to more northerlies during the roughly $5 \mathrm{~h}$ in agreement with the change of wind direction in the entrance region of the polar front jet, Fig. A3b, cf. Fig. 4d. From WP2 to the southernmost way point WP3 the wind direction became westerly. In this portion of the flight, $V_{H}$ increased further to about $30 \mathrm{~m} \mathrm{~s}^{-1}$. Fluctuations with amplitudes of up to $5 \mathrm{~m} \mathrm{~s}^{-1}$ are superimposed on the whole outbound and inbound flight legs.

Figure A3d reveals a difference in the potential temperature (equivalently also in absolute temperature as the aircraft flew on a constant pressure level) of more than $15 \mathrm{~K}$ between way points WP1 and WP3. Minimum temperatures were attained at the southernmost WP3 and around WP2 at about $53^{\circ} \mathrm{S}$. North of WP2, the temperature increased by about $3 \mathrm{~K}$ between the observations taken along the outbound and inbound legs, i.e., in a 5-h time period. This warming is related to the evolution of the weather system, especially, the advection of warm air, cf. Fig. 2b. Furthermore, and especially directly south and north of WP2 temperature fluctuations with amplitudes of less than $5 \mathrm{~K}$ interfere with the moderately changing temperature along the flight track. The GV flew in the lowermost stratosphere below $380 \mathrm{~K}$ (Holton et al. 1995) and crossed isentropes from about $360 \mathrm{~K}$ (north, WP1) to about $345 \mathrm{~K}$ (south, WP3).

The IFS predictions reproduce the overall meridional dependence of the observed flow variables quite well; however, deviations exist in regions where the real atmosphere was impacted by the polar front jets. Small-scale fluctuations are not present in the IFS data, especially the vertical wind is underestimated, Fig. A3a. The simulated temperature profiles follow the observed ones closely and the IFS captures the warming at around $51^{\circ} \mathrm{S}$ very well.

\section{REFERENCES}

Abdalla, S., L. Isaksen, P. A. E. M. Janssen, and N. Wedi, 2013: Effective spectral resolution of ECMWF atmospheric forecast models. ECMWF Newsletter, No. 137, Reading, United Kingdom, ECMWF, 19-22, https://doi.org/10.21957/rue4o7ac.

Alexander, M. J., and A. W. Grimsdell, 2013: Seasonal cycle of orographic gravity wave occurrence above small islands in the Southern Hemisphere: Implications for effects on the general circulation. J. Geophys. Res. Atmos., 118, 11589 11599, https://doi.org/10.1002/2013JD020526.

Alexander, S. P., K. Sato, S. Watanabe, Y. Kawatani, and D. J. Murphy, 2016: Southern Hemisphere extratropical gravity wave sources and intermittency revealed by a middle-atmosphere general circulation model. J. Atmos. Sci., 73, 13351349, https://doi.org/10.1175/JAS-D-15-0149.1.

Bacmeister, J. T., M. R. Schoeberl, L. R. Lait, P. A. Newman, and B. Gary, 1990: ER-2 mountain wave encounter over 
Antarctica: Evidence for blocking. Geophys. Res. Lett., 17, 81-84, https://doi.org/10.1029/GL017i001p00081.

Birner, T., 2006: Fine-scale structure of the extratropical tropopause region. J. Geophys. Res., 111, D04104, https://doi.org/ 10.1029/2005JD006301.

—, A. Dörnbrack, and U. Schumann, 2002: How sharp is the tropopause at midlatitudes? Geophys. Res. Lett., 29, 45-1-454, https://doi.org/10.1029/2002GL015142.

Carli, B., U. Cortesi, C. E. Blom, M. P. Chipperfield, G. D. Rossi, and G. Redaelli, 2000: Airborne Polar Experiment Geophysica Aircraft in Antarctica (APE-GAIA). SPARC Newsletter, No. $15,21-24$.

Clark, T. L., T. Hauf, and J. P. Kuettner, 1986: Convectively forced internal gravity waves: Results from two-dimensional numerical experiments. Quart. J. Roy. Meteor. Soc., 112, 899 925, https://doi.org/10.1002/qj.49711247402.

Cooper, W. A., and Coauthors, 2016: Characterization of uncertainty in measurements of wind from the NSF/NCAR Gulfstream V Research Aircraft. Tech. Note NCAR/TN528+STR, 161 pp., https://doi.org/10.5065/D60G3HJ8.

Denning, R. F., S. L. Guidero, G. S. Parks, and B. L. Gary, 1989: Instrument description of the airborne microwave temperature profiler. J. Geophys. Res. Atmos., 94, 16757-16765, https://doi.org/10.1029/JD094iD14p16757.

Dunkerton, T. J., 1984: Inertia-gravity waves in the stratosphere. J. Atmos. Sci., 41, 3396-3404, https://doi.org/10.1175/15200469(1984)041<3396:IWITS > 2.0.CO;2.

Dutton, J. A., 1976: The Ceaselees Wind. 1st ed. McGraw-Hill, 579 pp.

Eckermann, S. D., and Coauthors, 2018: High-altitude (0-100 km) global atmospheric reanalysis system: Description and application to the 2014 austral winter of the Deep Propagating Gravity Wave Experiment (DEEPWAVE). Mon. Wea. Rev., 146, 2639-2666, https://doi.org/10.1175/MWR-D-17-0386.1.

— J. D. Doyle, P. A. Reinecke, C. A. Reynolds, R. B. Smith, D. C. Fritts, and A. Dörnbrack, 2019: Stratospheric gravity wave products from satellite infrared nadir radiances in the planning, execution, and validation of aircraft measurements during DEEPWAVE. J. Appl. Meteor. Climatol., 58, 20492075, https://doi.org/10.1175/JAMC-D-19-0015.1.

Ehard, B., and Coauthors, 2017: Horizontal propagation of largeamplitude mountain waves into the polar night jet. J. Geophys. Res. Atmos., 122, 1423-1436, https://doi.org/10.1002/ 2016JD025621.

— S. Malardel, A. Dörnbrack, B. Kaifler, N. Kaifler, and N. Wedi, 2018: Comparing ECMWF high-resolution analyses with lidar temperature measurements in the middle atmosphere. Quart. J. Roy. Meteor. Soc., 144, 633-640, https://doi. org/10.1002/qj.3206.

Eliassen, A., and E. Palm, 1960: On the Transfer of Energy in Stationary Mountain Waves. I kommisjon hos Aschehoug, 23 pp.

Fritts, D. C., and Coauthors, 2016: The Deep Propagating Gravity Wave Experiment (DEEPWAVE): An airborne and groundbased exploration of gravity wave propagation and effects from their sources throughout the lower and middle atmosphere. Bull. Amer. Meteor. Soc., 97, 425-453, https://doi.org/ 10.1175/BAMS-D-14-00269.1.

Garcia, R. R., A. K. Smith, D. E. Kinnison, Á. de la Cámara, and D. J. Murphy, 2017: Modification of the gravity wave parameterization in the Whole Atmosphere Community Climate Model: Motivation and results. J. Atmos. Sci., 74, 275-291, https://doi.org/10.1175/JAS-D-16-0104.1.
Garfinkel, C. I., and L. D. Oman, 2018: Effect of gravity waves from small islands in the Southern Ocean on the Southern Hemisphere atmospheric circulation. J. Geophys. Res. Atmos., 123, 1552-1561, https://doi.org/10.1002/2017JD027576.

Gary, B. L., 1989: Observational results using the microwave temperature profiler during the Airborne Antarctic Ozone Experiment. J. Geophys. Res., 94, 11223-11231, https://doi. org/10.1029/JD094iD09p11223.

_ 2008: Mesoscale temperature fluctuations in the Southern Hemisphere stratosphere. Atmos. Chem. Phys., 8, 4677-4681, https://doi.org/10.5194/acp-8-4677-2008.

Gisinger, S., and Coauthors, 2017: Atmospheric conditions during the Deep Propagating Gravity Wave Experiment (DEEPWAVE). Mon. Wea. Rev., 145, 4249-4275, https://doi.org/10. 1175/MWR-D-16-0435.1.

Glatt, I., A. Dörnbrack, S. Jones, J. Keller, O. Martius, A. Müller, D. H. W. Peters, and V. Wirth, 2011: Utility of Hovmöller diagrams to diagnose Rossby wave trains. Tellus, 63A, 9911006, https://doi.org/10.1111/j.1600-0870.2011.00541.x.

Hauchecorne, A., and M.-L. Chanin, 1980: Density and temperature profiles obtained by lidar between 35 and $70 \mathrm{~km}$. Geophys. Res. Lett., 7, 565-568, https://doi.org/10.1029/ GL007i008p00565.

Hendricks, E. A., J. D. Doyle, S. D. Eckermann, Q. Jiang, and P. A. Reinecke, 2014: What is the source of the stratospheric gravity wave belt in austral winter? J. Atmos. Sci., 71, 15831592, https://doi.org/10.1175/JAS-D-13-0332.1.

Hersbach, H., and Coauthors, 2020: The ERA5 global reanalysis. Quart. J. Roy. Meteor. Soc., 146, 1999-2049, https://doi.org/10. 1002/qj.3803.

Hewson, T. D., 1998: Objective fronts. Meteor. Appl., 5, 37-65, https://doi.org/10.1017/S1350482798000553.

Hindley, N. P., C. J. Wright, N. D. Smith, and N. J. Mitchell, 2015: The southern stratospheric gravity wave hot spot: Individual waves and their momentum fluxes measured by COSMIC GPS-RO. Atmos. Chem. Phys., 15, 7797-7818, https:// doi.org/10.5194/acp-15-7797-2015.

_, - L. Hoffmann, T. Moffat-Griffin, and N. J. Mitchell, 2020: An 18-year climatology of directional stratospheric gravity wave momentum flux from 3-d satellite observations. Geophys. Res. Lett., 47, e2020GL089557, https://doi.org/10. 1029/2020GL089557.

Hogan, T. F., and Coauthors, 2014: The navy global environmental model. Oceanography, 27, 116-125, https://doi.org/10.5670/ oceanog.2014.73.

Holton, J. R., P. H. Haynes, M. E. McIntyre, A. R. Douglass, R. B. Rood, and L. P. Fister, 1995: Stratosphere-troposphere exchange. Rev. Geophys., 33, 403-439, https://doi.org/10.1029/ 95RG02097.

Höpfner, M., and Coauthors, 2006: MIPAS detects Antarctic stratospheric belt of NAT PSCs caused by mountain waves. Atmos. Chem. Phys., 6, 1221-1230, https://doi.org/10.5194/ acp-6-1221-2006.

Hoskins, B. J., and K. I. Hodges, 2005: A new perspective on Southern Hemisphere storm tracks. J. Climate, 18, 4108-4129, https://doi.org/10.1175/JCLI3570.1.

Hovmöller, E., 1949: The trough-and-ridge diagram. Tellus, 1, 62-66, https://doi.org/10.3402/tellusa.v1i2.8498.

Jiang, Q., J. D. Doyle, S. D. Eckermann, and B. P. Williams, 2019: Stratospheric trailing gravity waves from New Zealand. J. Atmos. Sci., 76, 1565-1586, https://doi.org/10.1175/JAS-D18-0290.1. 
Joyce, M., 2014: Bureau of Meteorology Macquarie Island Radiosonde Data, version 1.0. UCAR/NCAR-Earth Observing Laboratory, accessed 13 November 2020, https://doi.org/10. 5065/D66M357B.

Kruse, C. G., and R. B. Smith, 2015: Gravity wave diagnostics and characteristics in mesoscale fields. J. Atmos. Sci., 72, 43724392, https://doi.org/10.1175/JAS-D-15-0079.1.

,-- , and A. D. Nugent, 2016: NSF/NCAR GV HIAPER Energy and Momentum Flux Data, version 1.0. UCAR/ NCAR-Earth Observing Laboratory, accessed 13 November 2020, https://doi.org/10.5065/D6CF9NFX.

Madden, R. A., 2007: Large-scale, free Rossby waves in the atmosphere-An update. Tellus, 59A, 571-590, https://doi. org/10.1111/j.1600-0870.2007.00257.x.

Mahoney, M., and R. Denning, 2009: A state-of-the-art airborne microwave temperature profiler (MTP). Proc. 33rd Int. Symp. on the Remote Sensing of the Environment, Stresa, Italy, European Commission, https://archive.eol.ucar.edu/raf/ instruments/MTP/www/papers/33_ISRSE_SOTA_MTP_ Rev1.pdf.

Malardel, S., and N. P. Wedi, 2016: How does subgrid-scale parametrization influence nonlinear spectral energy fluxes in global NWP models? J. Geophys. Res. Atmos., 121, 53955410, https://doi.org/10.1002/2015JD023970.

— - - W. Deconinck, M. Diamantakis, C. Kuehnlein, G. Mozdzynski, M. Hamrud, and P. K. Smolarkiewicz, 2016: A new grid for the IFS. ECMWF Newsletter, No. 146, Reading, United Kingom, 23-28, https://doi.org/10.21957/zwdu9u5i.

Martius, O., C. Schwierz, and H. C. Davies, 2010: Tropopauselevel waveguides. J. Atmos. Sci., 67, 866-879, https://doi.org/ 10.1175/2009JAS2995.1.

McLandress, C., T. G. Shepherd, S. Polavarapu, and S. R. Beagley, 2012: Is missing orographic gravity wave drag near $60^{\circ} \mathrm{S}$ the cause of the stratospheric zonal wind biases in chemistryclimate models? J. Atmos. Sci., 69, 802-818, https://doi.org/10. 1175/JAS-D-11-0159.1.

Morgan, M. C., and J. W. Nielsen-Gammon, 1998: Using tropopause maps to diagnose midlatitude weather systems. Mon. Wea. Rev., 126, 2555-2579, https://doi.org/10.1175/15200493(1998)126<2555:UTMTDM > 2.0.CO;2.

Parish, T. R., and D. H. Bromwich, 1989: Instrumented aircraft observations of the katabatic wind regime near Terra Nova Bay. Mon. Wea. Rev., 117, 1570-1585, https://doi.org/10.1175/ 1520-0493(1989)117<1570:IAOOTK>2.0.CO;2.

Pautet, P.-D., M. J. Taylor, S. D. Eckermann, and N. Criddle, 2019: Regional distribution of mesospheric small-scale gravity waves during DEEPWAVE. J. Geophys. Res. Atmos., 124, 7069-7081, https://doi.org/10.1029/2019JD030271.

Pedlosky, J., 1987: Geophysical Fluid Dynamics.1st ed. Springer, 710 pp., https://doi.org/10.1007/978-1-4612-4650-3.

Pfister, L., S. Scott, M. Loewenstein, S. Bowen, and M. Legg, 1993: Mesoscale disturbances in the tropical stratosphere excited by convection: Observations and effects on the stratospheric momentum budget. J. Atmos. Sci., 50, 1058-1075, https://doi.org/10.1175/1520-0469(1993)050<1058:MDITTS >2. $0 . \mathrm{CO} ; 2$.

Plougonven, R., and F. Zhang, 2014: Internal gravity waves from atmospheric jets and fronts. Rev. Geophys., 52, 33-76, https:// doi.org/10.1002/2012RG000419.

— A. Hertzog, and M. J. Alexander, 2015: Case studies of nonorographic gravity waves over the Southern Ocean emphasize the role of moisture. J. Geophys. Res. Atmos., 120, 12781299, https://doi.org/10.1002/2014JD022332.
— , V. Jewtoukoff, A. de la Cámara, F. Lott, and A. Hertzog, 2017: On the relation between gravity waves and wind speed in the lower stratosphere over the Southern Ocean. J. Atmos. Sci., 74, 1075-1093, https://doi.org/10.1175/JAS-D-16-0096.1.

Plumb, R. A., and A. D. McEwan, 1978: The instability of a forced standing wave in a viscous stratified fluid: A laboratory analogue of the quasi-biennial oscillation. J. Atmos. Sci., 35, 1827-1839, https://doi.org/10.1175/1520-0469(1978) $035<1827$ :TIOAFS $>2.0$. CO;2.

Polichtchouk, I., R. Hogan, T. Shepherd, P. Bechtold, T. Stockdale, S. Malardel, S.-J. Lock, and L. Magnusson, 2017: What influences the middle atmosphere circulation in the IFS? ECMWF Tech. Memo. 809, 48 pp., https://doi.org/10. 21957/mfsnfv15o.

_ , and Coauthors, 2021: Stratospheric modelling and assimilation. ECMWF Tech. Memo. 877, 61 pp., https://doi.org/10. 21957/25hegfoq.

Preusse, P., A. Dörnbrack, S. D. Eckermann, M. Riese, B. Schaeler, J. T. Bacmeister, D. Broutman, and K. U. Grossmann, 2002: Space-based measurements of stratospheric mountain waves by CRISTA 1 . Sensitivity, analysis method, and a case study. J. Geophys. Res. Atmos., 107, 8178, https://doi.org/10.1029/ 2001JD000699.

Prusa, J. M., P. K. Smolarkiewicz, and R. R. Garcia, 1996: Propagation and breaking at high altitudes of gravity waves excited by tropospheric forcing. J. Atmos. Sci., 53, 2186-2216, https:// doi.org/10.1175/1520-0469(1996)053<2186:PABAHA > 2.0.CO;2. , and - 2003: An all-scale anelastic model for geophysical flows: Dynamic grid deformation. J. Comput. Phys., 190, 601622, https://doi.org/10.1016/S0021-9991(03)00299-7.

Randel, W. J., F. Wu, and P. Forster, 2007: The extratropical tropopause inversion layer: Global observations with GPS data, and a radiative forcing mechanism. J. Atmos. Sci., 64, 44894496, https://doi.org/10.1175/2007JAS2412.1.

Rapp, M., and Coauthors, 2021: SOUTHTRAC-GW: An airborne field campaign to explore gravity wave dynamics at the world's strongest hotspot. Bull. Amer. Meteor. Soc., 102, E871-E893, https://doi.org/10.1175/BAMS-D-20-0034.1.

Reed, R. J., and E. F. Danielsen, 1958: Fronts in the vicinity of the tropopause. Arch. Meteor. Geophys. Biokl., 11A, 1-17, https://doi.org/10.1007/BF02247637.

Renard, R. J., and L. C. Clarke, 1965: Experiments in numerical objective frontal analysis. Mon. Wea. Rev., 93, 547-556, https://doi.org/10.1175/1520-0493(1965)093<0547:EINOFA $>2$. 3.CO;2.

Sato, K., S. Tateno, S. Watanabe, and Y. Kawatani, 2012: Gravity wave characteristics in the Southern Hemisphere revealed by a high-resolution middle-atmosphere general circulation model. J. Atmos. Sci., 69, 1378-1396, https://doi.org/10.1175/ JAS-D-11-0101.1.

Schultz, D. M., and Coauthors, 2019: Extratropical cyclones: A century of research on meteorology's centerpiece. A Century of Progress in Atmospheric and Related Sciences: Celebrating the American Meteorological Society Centennial, Meteor. Monogr., No. 59, Amer. Meteor. Soc., 16.1-16.56, https://doi. org/10.1175/AMSMONOGRAPHS-D-18-0015.1.

Schumann, U., 2019: The horizontal spectrum of vertical velocities near the tropopause from global to gravity wave scales. $J$. Atmos. Sci., 76, 3847-3862, https://doi.org/10.1175/JAS-D-190160.1.

Shapiro, M. A., and D. Keyser, 1990: Fronts, jet streams and the tropopause. Extratropical Cyclones: The Erik Palmén Memorial Volume, C. W. Newton and E. O. Holopainen, Eds., 
Amer. Meteor. Soc., 167-191, https://doi.org/10.1007/978-1944970-33-8_10.

—, and Coauthors, 1999: A planetary-scale to mesoscale perspective of the life cycles of extratropical cyclones: The bridge between theory and observations. The Life Cycles of Extratropical Cyclones, M. A. Shapiro and S. Grønås, Eds., Amer. Meteor. Soc., 139-185, https://doi.org/10.1007/978-1935704-09-6_14.

Smith, R. B., B. K. Woods, J. Jensen, W. A. Cooper, J. D. Doyle, Q. Jiang, and V. Grubišić, 2008: Mountain waves entering the stratosphere. J. Atmos. Sci., 65, 2543-2562, https://doi.org/ 10.1175/2007JAS2598.1.

— , and Coauthors, 2016: Stratospheric gravity wave fluxes and scales during DEEPWAVE. J. Atmos. Sci., 73, 2851-2869, https://doi.org/10.1175/JAS-D-15-0324.1.

Tateno, S., and K. Sato, 2008: A study of inertia-gravity waves in the middle stratosphere based on intensive radiosonde observations. J. Meteor. Soc. Japan, 86, 719-732, https://doi.org/10. 2151/jmsj.86.719.

Thomas, C. M., and D. M. Schultz, 2019: What are the best thermodynamic quantity and function to define a front in gridded model output? Bull. Amer. Meteor. Soc., 100, 873-895, https://doi.org/10.1175/BAMS-D-18-0137.1.

Trenberth, K. E., 1991: Storm tracks in the Southern Hemisphere. J. Atmos. Sci., 48, 2159-2178, https://doi.org/10.1175/15200469(1991)048<2159:STITSH > 2.0.CO;2.

Tuck, A. F., 2008: Atmospheric Turbulence: A Molecular Dynamics Perspective. 1st ed. Oxford University Press, $184 \mathrm{pp}$.

- 2021: Perspective on aircraft in the stratosphere: 50 years from COMESA through the ozone hole to climate. Quart. J. Roy. Meteor. Soc., 147, 713-727, https://doi.org/10.1002/qj. 3958.

—, R. T. Watson, E. P. Condon, J. J. Margitan, and O. B. Toon, 1989: The planning and execution of ER-2 and DC8 aircraft flights over Antarctica, August and September 1987. J. Geophys. Res. Atmos., 94, 11181-11222, https://doi. org/10.1029/JD094iD09p11181.

— W. W. Brune, and R. S. Hipskind, 1997: Airborne Southern Hemisphere Ozone Experiment/Measurements for Assessing the Effects of Stratospheric Aircraft (ASHOE/MAESA): A road map. J. Geophys. Res. Atmos., 102, 3901-3904, https:// doi.org/10.1029/96JD02745.

UCAR/NCAR-Earth Observing Laboratory, 2015a: DEEPWAVE: Low Rate (LRT - 1 sps) Navigation, State Parameter, and Microphysics Flight-Level Data, version 1.2. UCAR/NCAR-
Earth Observing Laboratory, accessed 13 November 2020, https://doi.org/10.5065/D6J964D9.

— 2015b: Microwave Temperature Profiler, version 1.0. UCAR/NCAR-Earth Observing Laboratory, accessed 13 November 2020, https://doi.org/10.5065/D6H70D56.

—, 2016: NSF/NCAR GV HIAPER QC Dropsonde Data, version 3. UCAR/NCAR-Earth Observing Laboratory, accessed 13 November 2020, https://doi.org/10.5065/D6XW4GTB.

Vallis, G. K., 2017: Atmospheric and Oceanic Fluid Dynamics: Fundamentals and Large-Scale Circulation. 2nd ed. Cambridge University Press, 964 pp., https://doi.org/10.1017/ 9781107588417.

Wang, S., F. Zhang, and C. Snyder, 2009: Generation and propagation of inertia-gravity waves from vortex dipoles and jets. J. Atmos. Sci., 66, 1294-1314, https://doi.org/10.1175/ 2008JAS2830.1.

Wedi, N. P., 2014: Increasing horizontal resolution in numerical weather prediction and climate simulations: Illusion or panacea? Philos. Trans. Roy. Soc. London, 372A, 20130289, https://doi.org/10.1098/rsta.2013.0289.

— , and P. K. Smolarkiewicz, 2006: Direct numerical simulation of the Plumb-McEwan laboratory analog of the QBO. $J$. Atmos. Sci., 63, 3226-3252, https://doi.org/10.1175/JAS3815.1.

Wei, J., and F. Zhang, 2014: Mesoscale gravity waves in moist baroclinic jet-front systems. J. Atmos. Sci., 71, 929-952, https:// doi.org/10.1175/JAS-D-13-0171.1.

Williams, B. P., 2015: NSF/NCAR GV HIAPER Uplooking Rayleigh Lidar Data, version 0.1 [PRELIMINARY]. UCAR/ NCAR-Earth Observing Laboratory, accessed 13 November 2020, https://data.eol.ucar.edu/dataset/379.034.

Wirth, V., M. Riemer, E. K. M. Chang, and O. Martius, 2018: Rossby wave packets on the midlatitude waveguide-A review. Mon. Wea. Rev., 146, 1965-2001, https://doi.org/10. 1175/MWR-D-16-0483.1.

Žagar, N., D. Jelić, M. Blaauw, and P. Bechtold, 2017: Energy spectra and inertia-gravity waves in global analyses. J. Atmos. Sci., 74, 2447-2466, https://doi.org/10.1175/JAS-D-16-0341.1.

Zhang, F., S. E. Koch, C. A. Davis, and M. L. Kaplan, 2000: A survey of unbalanced flow diagnostics and their application. Adv. Atmos. Sci., 17, 165-183, https://doi.org/10.1007/s00376000-0001-1.

— C. A. Davis, M. L. Kaplan, and S. E. Koch, 2001: Wavelet analysis and the governing dynamics of a large-amplitude mesoscale gravity-wave event along the East Coast of the United States. Quart. J. Roy. Meteor. Soc., 127, 2209-2245, https://doi.org/10.1002/qj.49712757702. 